\title{
The Effects of Fatigue Cracks on Fastener Loads during Cyclic Loading and on the Stresses Used for Crack Growth Analysis in Classical Linear Elastic Fracture Mechanics Approaches
}

\author{
Claudiu Gudas \\ QinetiQ Australia Pty Ltd., Melbourne, Australia \\ Email:CGudas@Qinetiq.com.au
}

How to cite this paper: Gudas, C. (2020) The Effects of Fatigue Cracks on Fastener Loads during Cyclic Loading and on the Stresses Used for Crack Growth Analysis in Classical Linear Elastic Fracture Mechanics Approaches. Materials Sciences and Applications, 11, 505-551. https://doi.org/10.4236/msa.2020.117035

Received: June 5, 2020

Accepted: July 24, 2020

Published: July 27, 2020

Copyright () 2020 by author(s) and Scientific Research Publishing Inc. This work is licensed under the Creative Commons Attribution International License (CC BY 4.0).

http://creativecommons.org/licenses/by/4.0/

(c) (i) Open Access

\begin{abstract}
High strength threaded fasteners are widely used in the aircraft industry, and service experience shows that for structures where shear loading of the joints is significant, like skin splices, fuselage joints or spar caps-web attachments, more cracks are initiated and grow from the edges of the fastener holes than from features like fillets radii and corners or from large access holes. The main causes of this cracking are the stress concentrations introduced by the fastener holes and by the threaded fasteners themselves, with the most common damage site being at the edge of the fastener holes. Intuitively, it is easy to visualize that after the crack initiation, during the growth stages, some of the load transferred initially by the fastener at the cracked hole will decrease, and it will be shed to the adjacent fasteners that will carry higher loads than in uncracked condition. Using currently available computer software, the method presented in this paper provides a relatively quick and quantitatively defined solution to account for the effects of crack length on the fastener loads transfer, and on the far field and bypass loads at each fastener adjacent to the crack. At each location, these variations are determined from the 3-dimensional distribution of stresses in the joint, and accounting for secondary bending effects and fastener tilt. Two cases of a typical skins lap splice with eight fasteners in a two rows configuration loaded in tension are presented and discussed, one representative for wing or fuselage skins configurations, and the second case representative for cost effective laboratory testing. Each case presents five cracking scenarios, with the cracks growing from approx. 0.03 inch to either the free edge, next hole or both simultaneously.
\end{abstract}




\section{Keywords}

Fasteners Load Transfer Functions, Fatigue Life, Multi-Site Fatigue Damage, Fasteners Modelling, Effects of Crack Length on Adjacent Fasteners, Secondary Bending, Crack Growth Analysis

\section{Introduction}

With the rise of fracture mechanics, the management and certification of safety critical or economically significant structures has switched towards crack propagation models using linear elastic fracture mechanics (LEFM) which correlates parameters like crack tip stress intensity factor to its growth rate. At the same time, new stronger materials have led to higher working stresses and, in many situations, having higher crack growth rates, so once initiated, the duration to a critical size is reduced [1].

High strength threaded fasteners are widely used in the aircraft industry, and service experience shows that for structures where shear loading of the joints is significant, like skin splices, fuselage joints or spar caps-web attachments, more cracks are initiated and grow from the edges of the fastener holes than from features like fillets radii and corners or from large access holes. The main causes of this cracking, particularly in the case of single shear joints, are the stress concentrations introduced by the fastener holes and by the threaded fasteners themselves with the most common damage site being at the edge of the fastener holes.

These types of joints have been addressed extensively by researchers and engineers using experimental and analytical techniques (e.g. [2] [3] [4] [5]), and two major steps, with their desired methods and data, have been identified for the analysis of the fatigue performance of fastened joints. These steps are:

- The calculations of the force distributions in the joint, with methods and data to account for fastener flexibility, eccentricities in the load paths and the support from surrounding structure, and

- The calculation of the fatigue quality, which requires the influence from the bypass loads, the loads transferred by the fasteners, the moments due to eccentricities and the effects of bearing and friction interplay to be known for all the materials, hole quality and fastener types in the joint.

A systematic review of some approaches used for fatigue analysis is presented by E. Jarfall in [6]. In reference [7], the same author defines a stress severity factor concept, and shows that both the far field and bearing contact loads have a significant influence on the fatigue strength of a shear joint. Methods and test data for shear and tension joints have been developed by many major aircraft manufacturers and/or research organizations. Some are published in the technical literature [8]-[13]. In all these methods, a clear distinction is made between hole filling rivets and threaded fasteners.

In a typical LEFM crack growth analysis, the effects of crack length and the in- 
fluence of nearby features like free edges, other holes, cracks and sometimes, the pin of the fastener, are accounted for by the use of geometry functions, mostly known as "the beta functions". The load transferred at the fastener is assumed to remain constant as one crack grows to the next nearby feature and, after that, it is often assumed that there is no more load transfer through that fastener.

Intuitively it is easy to visualize that after the crack initiation, during the growth stages, some of the load transferred initially by the fastener at the cracked hole will decrease, and it will be shed to the adjacent fasteners that will carry higher loads than in the uncracked condition. Concurrently with the changes in the load transfers at the fasteners, the bypass and even the far field loads will have to change to maintain the loads balance.

Using currently available computer software, the method presented in this paper provides a relatively quick and quantitatively defined solution to account for the effects of crack length on the fastener loads transfer at each fastener adjacent to the crack in a single shear loaded joint.

Two cases of a typical skins lap splice with eight fasteners in a two rows configuration loaded in tension are presented and discussed. The first case is representative for wing or fuselage skins and the second case is representative for cost effective laboratory testing. Both models present five cracking scenarios, with the cracks growing from approximately 0.03 inch to either the free edge, the next hole or both simultaneously.

The approach enables development of simple tabular/look up parametric functions for updating the reference stresses used with the "beta functions".

\section{Method Outline and Verification Details}

\subsection{Brief Overview of the Fastener Modelling Approach and Typical Results}

Contemporary finite element packages like Nastran or MSC Marc, Abaqus or Ansys have incorporated dedicated methods and/or elements to enable the modelling and evaluation of the local stress/strain fields at and around the pre-tensioned fasteners. The methods available involve splitting of the fastener shank in two parts and the "shortening" of the shank with a set of unidirectional multipoint constraint (MPC) equations until the desired pretension force is reached. After that the two parts are "locked" with the shortening remaining fixed while the other loads are being applied. A brief summary of this approach as used in MSC Nastran [14] is presented in Figure 1.

For all subsequent load cases, the control grid is fixed in all three directions ( 1 , $2,3)$. This approach is very effective when the fasteners are subject to separating tensile forces and when the components remain relatively fixed in operation, with no lateral loads requiring the fasteners to sustain shear forces. It is not compatible with large displacements nor with fasteners loaded in shear.

Another fastener modelling technique, introduced in reference [15], maintains the continuity of the pin without changing its installation clearance (does 


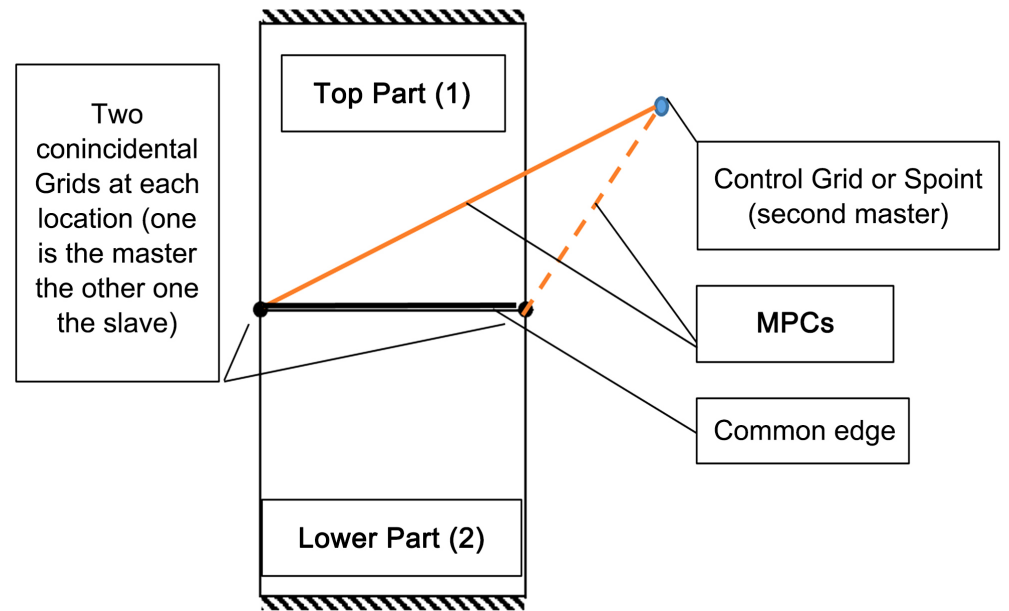

(a)

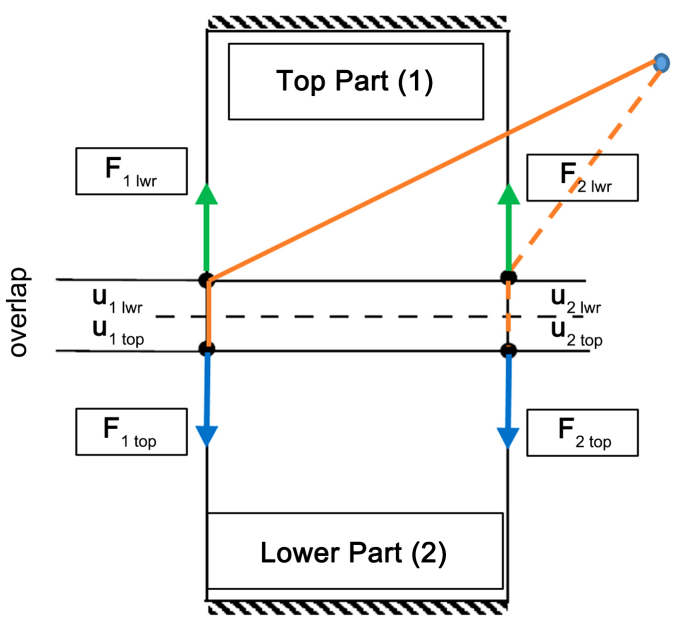

(b)

Figure 1. Split-pin methods available in MSC Nastran (explicit MPC or in BOLT formulations). (a) For each pair of coincidental points the MPC impose the following constraint equations on the model: $u_{l w r}-u_{\text {top }}-u_{\text {control }}=0$; (b) In these implementations the control grid is prevented to move in directions normal to the axis of the bolt by single points constraints (e.g. in dir. 1, 2).

not use thermal contraction ${ }^{1}$ ) and it is compatible with both large parts displacements and with the transfer of shear forces. For this approach, the bolt pin is kept continuous and a separate mesh is used for the nut. After the pretension step, the bolt and the nut are glued together along the common thread area and this condition is kept for all the subsequent loading steps. The initial pretension is introduced in the first step through two RBE3 elements, one acting on the pin shank and the other on the nut. A very short overview of this technique with typical results relevant to pretension and bearing loads and to the separation threshold [8] is shown in Figure 2.

${ }^{1}$ The use of thermal contraction for pretension would require a stiffness based analysis, similar to that presented in [8] for each joint configuration to establish the temperature differential $\Delta \mathrm{T}$ and it is not suitable when operational thermal considerations are of importance to structural or fatigue strength. 

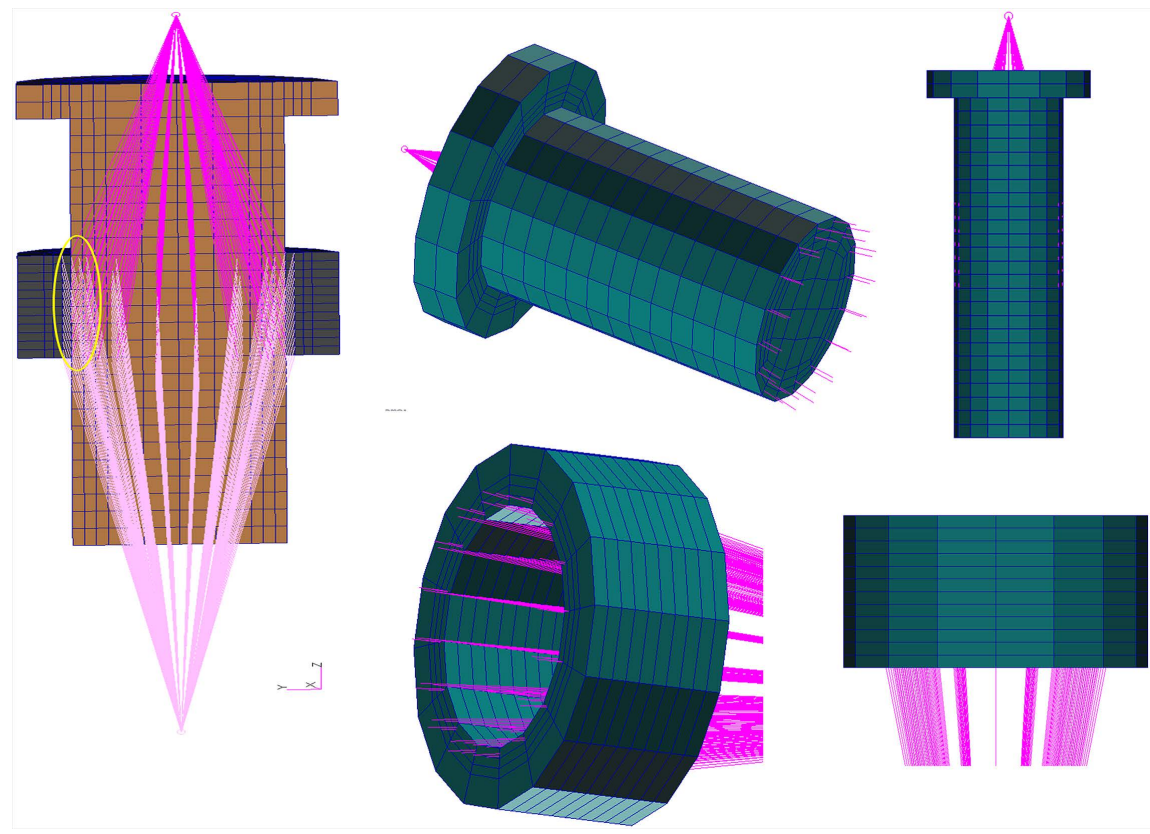

(a)

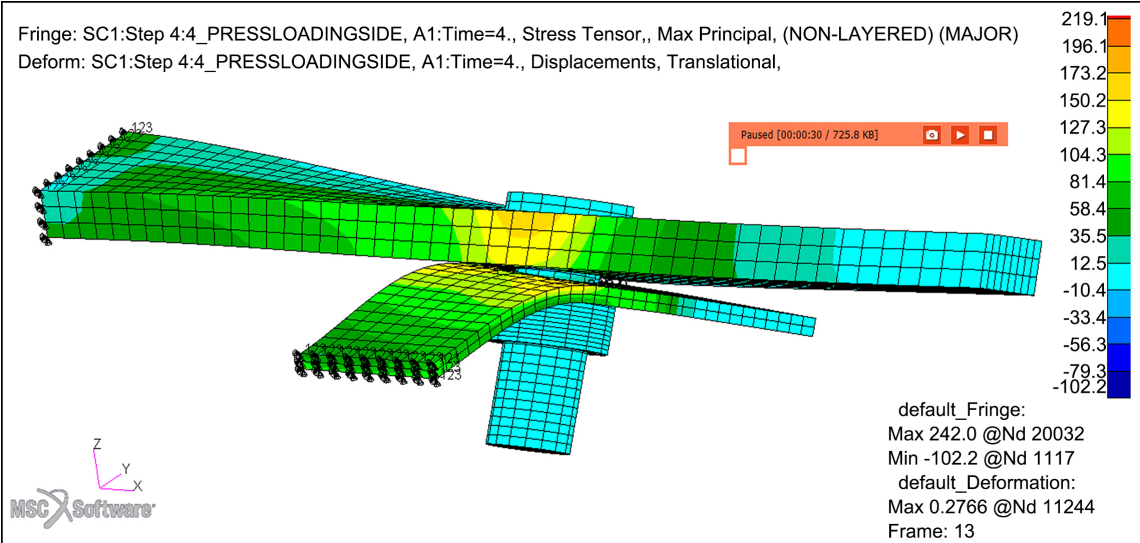

(b)

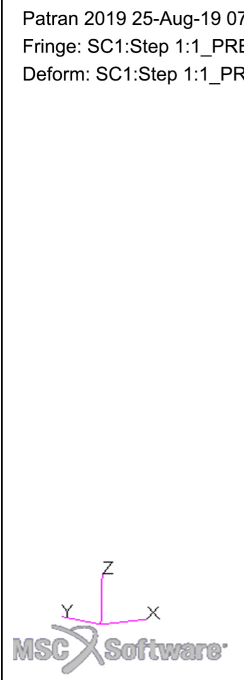

Patran 2019 25-Aug-19 07:36:05

Fringe: SC1:Step 1:1_PRETENSION (200N), A1:Time=1., Stress Tensor,, Max Principal, (NON-LAYERED) (MAJOR)

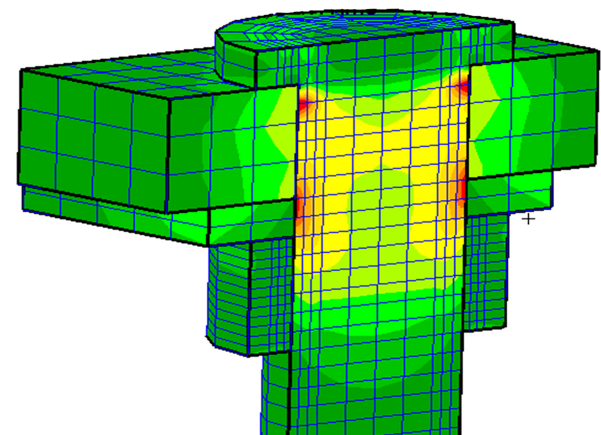

default Fringe: Max 56.93 @Nd 1018 Min -3.71@Nd 4776 default_Deformation: Max 0.0015 @Nd 520

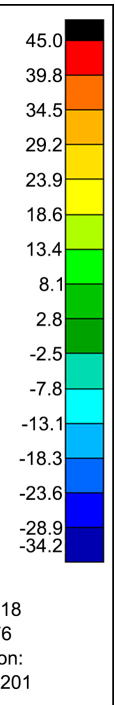

(c) 


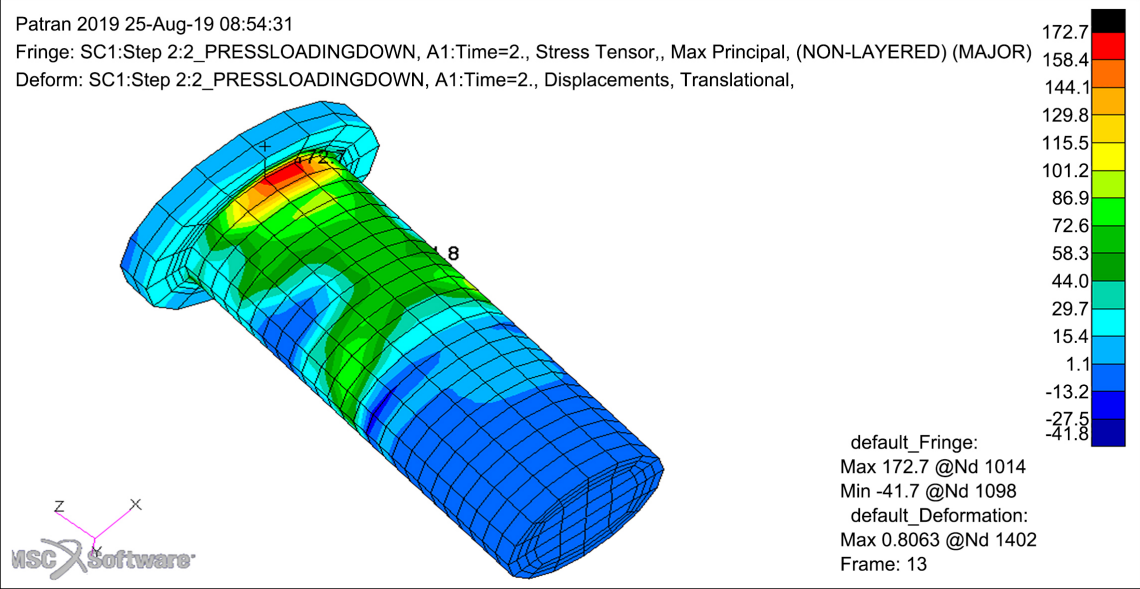

(d)

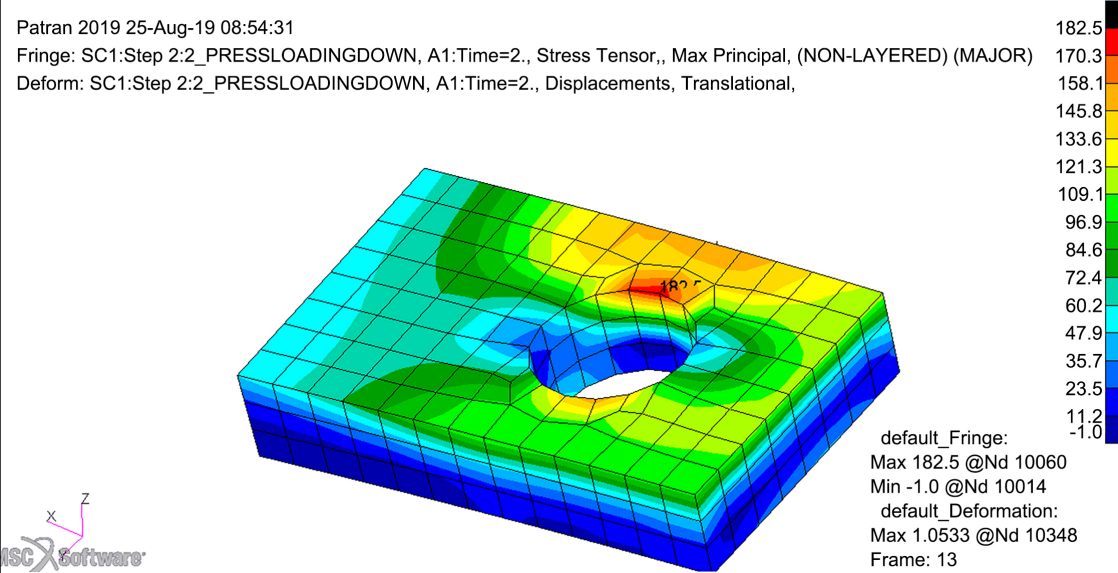

(e)

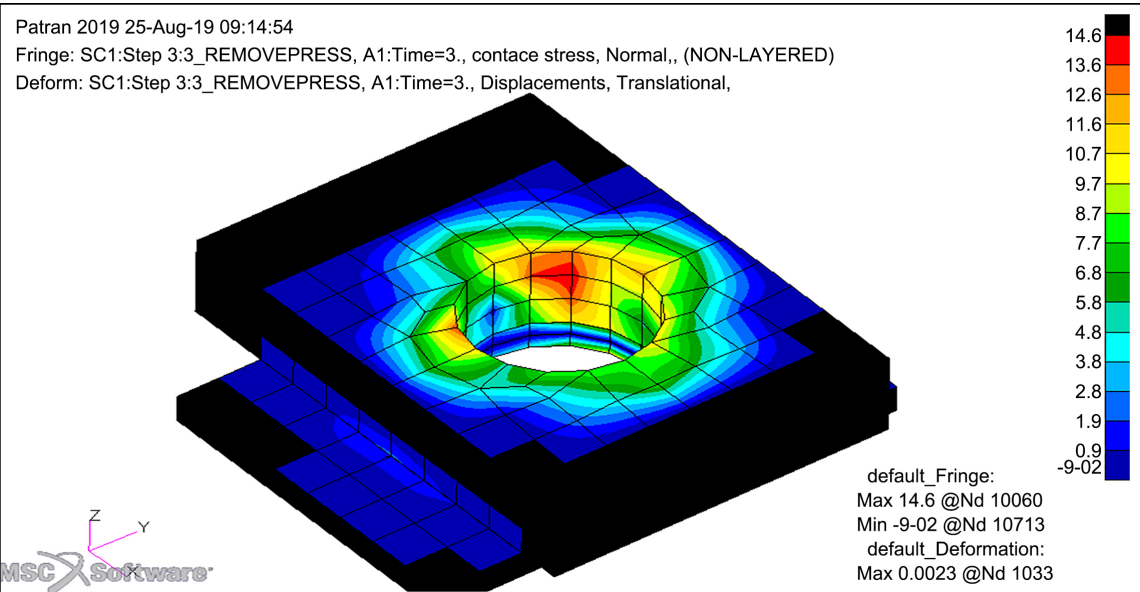

(f)

Figure 2. Definition of RBE3 for pretension and sample of typical results plots in Patran (Stresses in MPa, Deformations in $\mathrm{mm}$ ) (Deformations. true scale; Scale Factor 25:1). (a) RBE3 for bolt and for the nut (used only in the pre-tension step with the loads applied to the dependent nodes); (b) Joint complexly loaded above the separation threshold [8]. True deformation, scale factor 1:100; (c) Max principal stresses in part after pretension; (d) Max principal stresses in pin at full load; (e) Max principal stresses at hole for full load; (f) Contact stresses after removal of service load. 


\subsection{Method Outline}

\subsubsection{Geometry and FEA Representations}

The fastener modelling technique of reference [15] was used for a typical lap splice with eight fasteners in a two rows configuration, loaded in tension only. The analysis was done for two distinct cases, one representative for wing or fuselage skins marked as model $C$ and the second case representative for cost effective laboratory testing and marked as model $D$.

Both models are identical in terms of geometry, materials, fixed end supports and applied loads. The difference between models is in the boundary conditions applied along the lateral edges, and they are shown in Figure 3.

For the aircraft configuration, the long edges are fixed in direction 1 (Ox) to approximate the constraint imposed by the adjacent structure, whereas model D represents a typical coupon testing configuration where the lateral edges are free.

At the left end, both models have constraints in all three directions and for rotations around $\mathrm{Ox}$ (not. 1, 2, 3, 4 in Nastran). At the right edge, where the tension loads are applied in direction $\mathrm{Oy}$, the constraints are in 1 and 3 (Ox \& Oz). This simulates either the rest of the skin on model $\mathrm{C}$ or the jaws of the loading machine in model D.

The external load was applied in four increments of $0.5 \%, 10 \%, 90 \%$ and $100 \%$, is $278.8 \mathrm{lbf}(1240 \mathrm{~N})$, corresponding to $63 \mathrm{lbf} / \mathrm{in}(11.03 \mathrm{~N} / \mathrm{mm})$ running load or to a uniform gross area tension stress of $1.0 \mathrm{ksi}(6.895 \mathrm{MPa})$ in the top plate.

The FEA analysis was conducted using shell elements located at the mid planes of the two plates in Nastran solution 400 with non-linear material and
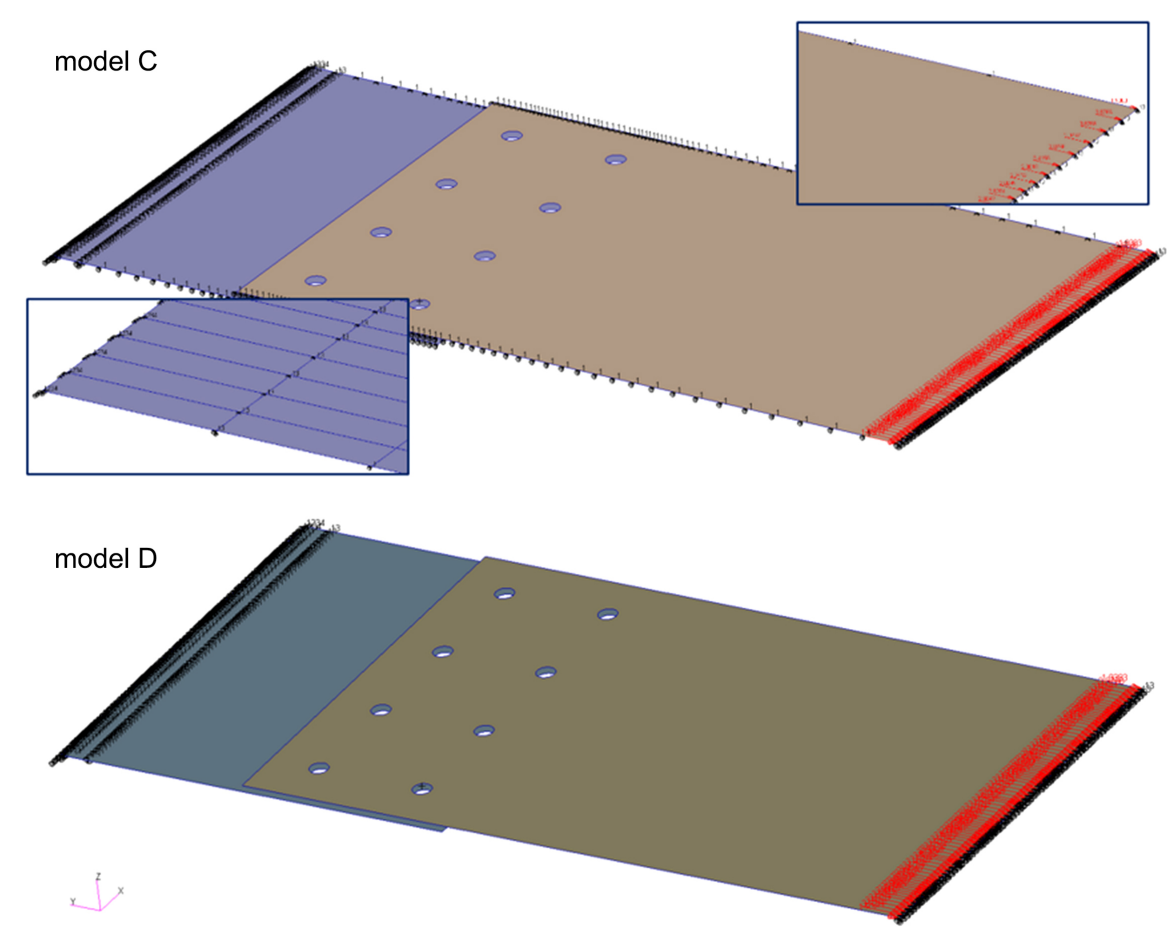

Figure 3. Diagram of loads application and boundary conditions. 
advanced formulation (large strain) for the two plates and, for the fasteners, solid elements with a linear material formulation for steel.

The geometry, materials and the specification for fasteners (including the close tolerance values ${ }^{2}$ ) are defined in Figure 4. In the same figure is shown the numbering used for the fastener holes and/or the fastener pins.

In Figure 5 is shown the meshing pattern used. The crack length increment used was defined by the sizes of the edges of the shell elements along the Ox axis. All units for the input/output were in the imperial system ( $l b f$, in, psi, lbf/in etc.).

For each fastener, four contact bodies were defined (Body_Pin1_Head, Body_Pin1_Thread, Body_Nut1_Head, Body_Nut1_Thread, etc.) and one contact body for each of the plates. Both models contained 21 contact zones defined for the initial set-up and setting in position, and 41 contact zones for the loading stage. With the exception of the contacts between the threads of fasteners and collars (glued), the friction was not included. This is because for conservatism, the friction is neglected in the structural analysis of aircraft structures. For the same reason the data in this paper does not include the initial pretension. Although the effects of pretension are significant for the crack initiation and growth, the aim of this paper is to address and quantify the effects of crack length on the forces (and stresses) used in popular current crack growth analysis programs.

The contacts at the threads of pins and collars were "glued" at the initial set-up (with the card BCTABL1 set to zero).

\subsubsection{Cracking Configurations Evaluated and Software Used for Pre and Post Processing}

Both models were evaluated for five cracking scenarios, with the cracks growing from approx. 0.03 inch to either the free edge, to the next hole or both simultaneously. The five configurations considered are shown in Table 1. In these FEA representations all the cracks are through thickness cracks

The FEA solver: MSC Nastran 2019SF1, running SOL 400. The generation of the input data decks and the shell batch-run command files was conducted using a combination of VBA subroutines in MS Excel 2019, UltraEditv26 from IDM Computer Solution for text editing and PTC Mathcad 5.0 and Prime 6.

The generation of Patran databases, the first stage of post processing and the extraction of results for each of the runs were conducted using dedicated PCL session files. The second stage of post processing contained the analysis and systematization of the results followed by data reduction for interpretation and generation of several levels of summary tables and graphs. It was conducted in batch mode for both models in Excel using VBA subroutines.

The following results were extracted and post processed for each crack length in all configurations of Table 1:

${ }^{2}$ In close tolerance the hole sizes are defined by the standardized Transition Fit classification for steel bolts in aluminium structure or by the Close Ream classification for steel bolts in steel or Ti structures. In the cases presented the fit between the pin and the hole in the FEA models was a clearance of $0.002 "$ in diameter. 
Table 1. Crack configurations evaluated and summary of post process results for each model.

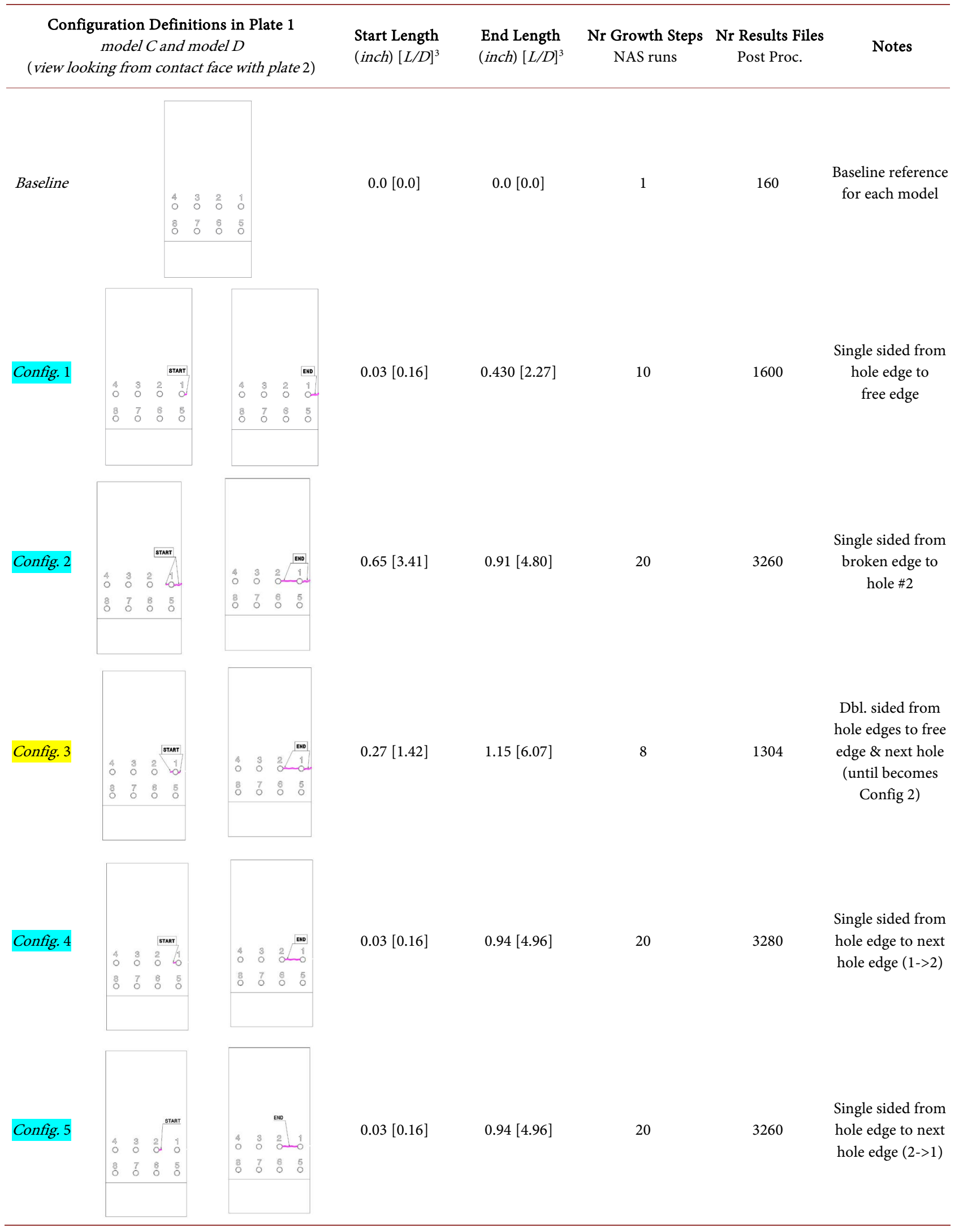

1) Single sided crack; 2) Double Sided crack; 3) All crack lengths rounded to two decimal places. 

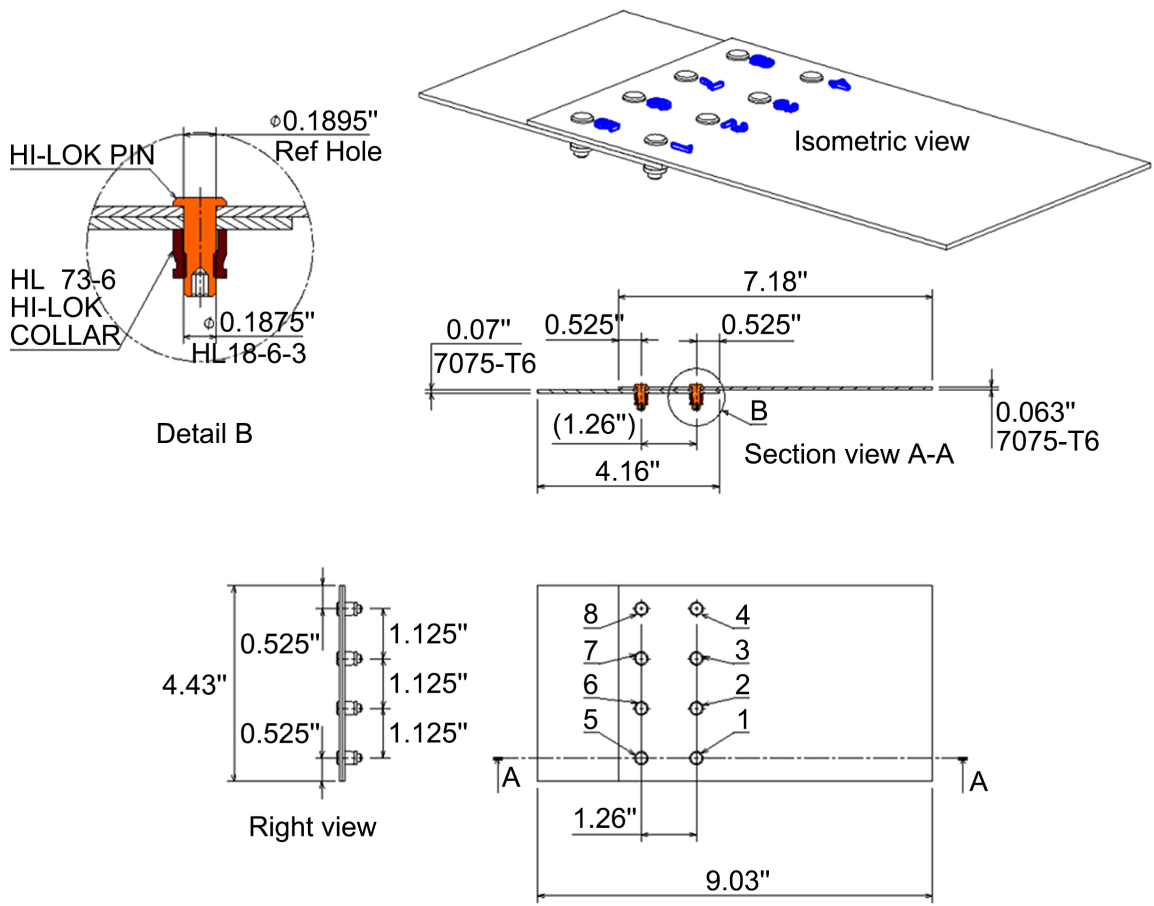

Front view

Figure 4. Geometry, materials and fasteners specifications.

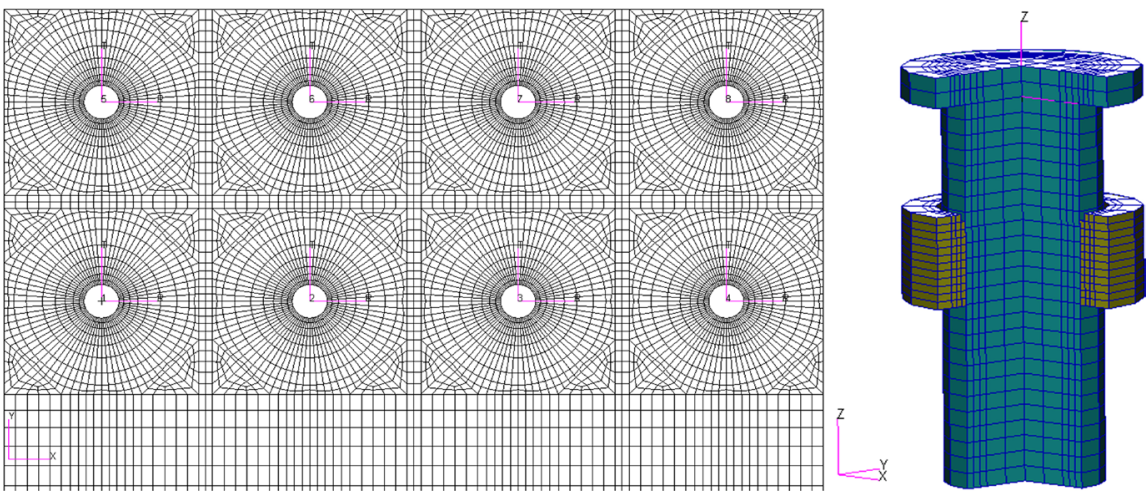

Figure 5. Illustration of the meshing patterns.

- Fastener loads: derived from the summation of Grid Point Balance outputs on all the nodes at the hole edges (outputs strictly in the basic coordinate system CID 0). The loads were extracted at all fasteners in the top plate (P1) and the lower plate (P2). At the edges of each hole there are 80 equally spaced nodes (Figure 6(a));

- Contact forces normal to the pins. these forces were derived from the Partran's outputs for "Resultants Contact Forces", this time at the pin nodes. There are 12 equally spaced layers of elements on each pin. The purpose of extracting the pin loads was to evaluate the distribution of the bearing forces at each hole at various depth levels of the plates. Figure $6(\mathrm{~b})$ shows the 12 layers in the pin and the location of the upper and lower plate shells. Layer 1 is located immediately under the fastener head while layers 9 to 12 are inside 


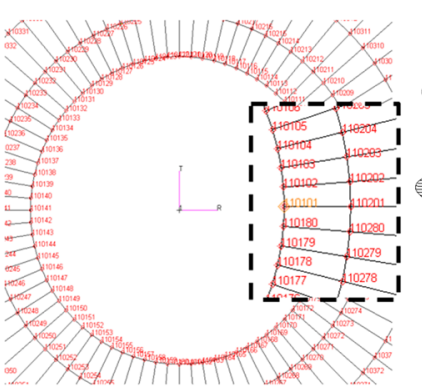

(a)

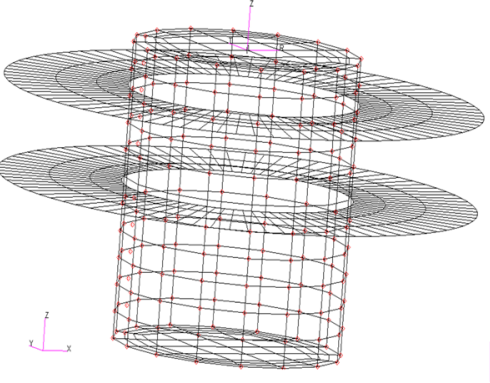

(b)

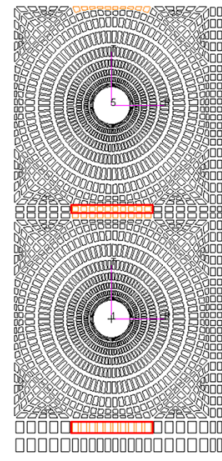

(c)

Figure 6. Exemplification of nodes and elements used in post processing. (a) for fastener loads at holes in panels P1 and P2; (b) for load variation of through the thickness of the holes in P1 and P2; (c) for far-field and bypass running loads and shear flows and stresses at the outer surfaces.

the collar. The separation plane between the two plates is inside layer no. 5, and therefore simple interpolations were necessary for the estimates of the contact forces in the plates at the separation plane.

- Far field and bypass running loads $s^{3}$ and shear flows. were extracted for each hole at the centroids of specified elements as $f_{x}, f_{y}$ and $q_{x y}$ in CID 0. These running loads were extracted only for the upper plate P1 using pre-defined bands of 10 elements ahead and after each fastener hole. These bands are located at approximatively two hole diameters away from the hole edges and they have a width of similar size (i.e. $0.435 ")$ perpendicular to the load direction and are symmetrical about the axes of the holes. The locations and width were chosen for their convenience of installing strain gauges during laboratory testing and because at these locations the secondary bending effects are representative for averaging and interpretation. In Figure 6(c) the elements used for extraction of far field running loads are highlighted and bordered by a red rectangle. For bypass, the elements used are only highlighted.

- Far field and bypass element centroidal stresses were extracted for the upper and lower surfaces of P1 at each hole, as $\sigma_{x}, \sigma_{y}$ and $\tau_{x y}$ strictly in CID 0 , as max-min principal invariants and as Tresca/max shear. These stresses were "averaged" for the band widths as described in section 3.3.

To keep uniformity with the bolt constant ${ }^{4} /$ fastener flexibility ${ }^{5}$ concepts of Tate and Rosenfeld/L. Jarfall, the traditional form of the gross area stress

\footnotetext{
${ }^{3}$ The axial running loads were defined as the distributed axial force acting per unit of length (analogue to the classical definition of shear flow) given by the ratio between the sum of the axial forces of the ten elements and their total length in direction Ox. Similar for lateral running loads.

${ }^{4}$ Bolt constant was defined as a linear approximation of the total displacement caused by fastener deformation, fastener tilt and the deformation of the fastener holes (all three deformations are nonlinearly dependent on the applied load). There are several semi-empirical formulae used in the industry for its calculations. It is commonly known as the "fastener flexibility constant" and is used to approximate the fastener loads in a $1 \mathrm{D}$ beam idealization.

${ }^{5}$ Fastener flexibility associates its displacement to the unit force of fastener load and does not imply that fastener flexibility would be a constant.
} 
$\left(\sigma_{\text {gross_oy }}\right)$ was also briefly evaluated as an alternative form for the interpretation of the results.

\section{Results}

\subsection{Effect of Crack Configuration and Crack Length on the Forces Transferred by the Fasteners}

For splices in tension, there is a distinct difference in the patterns of fastener load redistributions as functions of crack length between the single tip and double tip cracks. Also, the lateral restraints play a role in the way the loads from the fastener at the cracked hole are shed to adjacent fasteners. However, regardless of configuration and crack length, the variations of fastener loads are $\pm 10 \%$. Generally the fastener at the cracked hole becomes less loaded and the adjacent fasteners in same row experience load increases. The load shedding extends also to the fasteners in the second row, but the absolute changes in values are smaller. An envelope of percent variations from the baseline values is presented in Table 2.

The most significant observations from an engineering perspective are:

- For a single sided crack initiating from an edge hole and growing towards the free edge (Config. 1), the fastener load is at its minimum value just before the crack breaks though the end ligament. When the crack breaks through, the load at that fastener returns close to its value in baseline configuration. Noting that the fastener head and the collar continue hold the two plates together, this behavior was reconciled by considering the end supports provided by the plate for this fastener which, during the growth stage, are analogous to a beam with both edges restrained, and after breakthrough, the support configuration is single sided ("the hook effect"). As the ligament at the free edge breaks, the bypass loads in the plate at the other side of the cracked hole increase and the load transferred by fastener \#1 increases slightly over its baseline value.

- If, after the break through, a new crack initiates at the other side of the hole and grows inwards as in Config 2, the variations in fastener loads are similar to those in Config 1 but, after the crack breaking into the second hole, the loads at both fasteners remain below their baseline values.

- For one tip cracks initiating from holes remote from the free edges, the variations in the fastener loads are of similar ratios and, after the fracture of the ligament, the hook effects are smaller with both fasteners carrying between $90 \%$ and $95 \%$ of their initial load.

- The largest reductions in the fastener loads are for double sided cracks. After braking one or two ligaments the fastener at the cracked hole carries $20 \%$ less load than in baseline configuration. This load remains unchanged until a new crack starts to grow.

${ }^{6}$ Another visualization analogy may be made with a lug, when the crack breaks through one side, the loads are reacted in bending and shear like in a hook. 
Table 2. Envelopes of variations in fastener loads (\% of baseline values) (Row 1: fasteners 1 - 4; Row 2: fasteners 5 - 8).

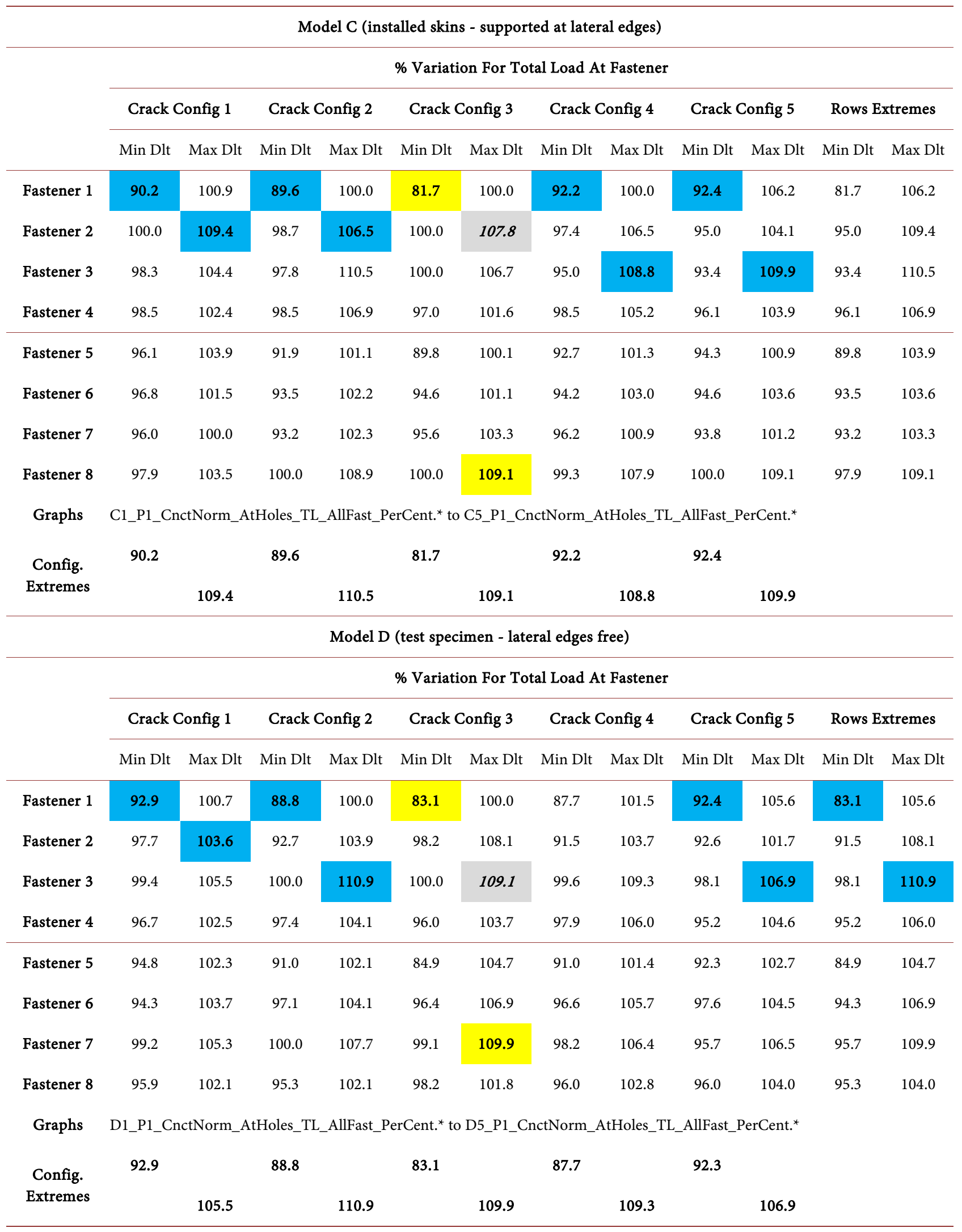

Single Sided Crack; $\quad$ : Double Sided Crack. 
- The functions of variation in the fastener loads are dependent on the cracking configuration, the crack length and the restraint conditions of the plates in the joint, but they are relatively easy to develop and represent as graphs or lookup tables for further use in engineering quantitative evaluations in both force values and/or in parametric format. The curves developed for the models and crack configurations discussed here are presented in Figure 7 to Figure 11.

For quick reference the final baseline fastener loads are shown in Table 3. All values are in CID 0 (the coordinate system shown in Figure 6(c)).

\subsection{Effect of Crack Length on the Through Thickness Distribution of Bearing Forces between the Fastener Pins and the Hole Surfaces}

The results for the bearing distributions at the pin-hole interfaces account for the fastener load transfer, for the eccentricity of the joint (secondary bending), bolt tilting and for the effects of the contact forces between the pins and the surfaces of the holes in the plates and between the fastener heads and the plates, albeit the fact that $2 \mathrm{D}$ elements located at midplane were used for the plates. They are however preliminary results because the pretension clamping forces, friction and the factors influencing the tractions forces between the faying surfaces (like cladding, sealants, etc) have not been included.

Table 3. Baseline fastener loads C model.

\begin{tabular}{|c|c|c|c|c|c|c|c|c|c|}
\hline \multicolumn{10}{|c|}{ Baseline All Fastener Loads - Model C } \\
\hline Model & $\mathrm{C}$ & Pin/Hole & Pin/Hole & Pin/Hole & Pin/Hole & Pin/Hole & Pin/Hole & Pin/Hole & Pin/Hole \\
\hline Panel & P1 & 1 & 2 & 3 & 4 & 5 & 6 & 7 & 8 \\
\hline Hole Dia & (in) & 0.1895 & 0.1895 & 0.1895 & 0.1895 & 0.1895 & 0.1895 & 0.1895 & 0.1895 \\
\hline Crack Length@ Current Fastener & (in) & 0.000000 & 0.000000 & 0.000000 & 0.000000 & 0.000000 & 0.000000 & 0.000000 & 0.000000 \\
\hline Load.Magnit $\left(\mathrm{X}^{\wedge} 2+\mathrm{Y}^{\wedge} 2+\mathrm{Z}^{\wedge} 2\right)^{\wedge} 0.5$ & $(I b f)$ & 33.5 & 34.8 & 35.4 & 34.6 & 34.5 & 35.8 & 36.6 & 33.3 \\
\hline Ox Total Load & $(I b f)$ & -0.2 & -0.2 & -0.3 & 0.2 & 0.2 & -0.2 & -0.2 & 0.9 \\
\hline Oy Total Load & $(I b f)$ & 33.5 & 34.8 & 35.4 & 34.6 & 34.5 & 35.8 & 36.6 & 33.3 \\
\hline Oz Total Load & $(I b f)$ & 6.6 & 5.7 & 5.8 & 4.8 & 2.4 & 2.6 & 2.6 & 2.4 \\
\hline Angle & $(\mathrm{deg})$ & 90.40 & 90.38 & 90.41 & 89.62 & 89.70 & 90.38 & 90.33 & 88.47 \\
\hline
\end{tabular}

Table 4. Baseline fastener loads (D Model).

\begin{tabular}{|c|c|c|c|c|c|c|c|c|c|}
\hline \multicolumn{10}{|c|}{ Baseline All Fastener Loads - Model D } \\
\hline Model & $\mathrm{D}$ & Pin/Hole & Pin/Hole & Pin/Hole & Pin/Hole & Pin/Hole & Pin/Hole & Pin/Hole & Pin/Hole \\
\hline Panel & $\mathrm{P} 1$ & 1 & 2 & 3 & 4 & 5 & 6 & 7 & 8 \\
\hline Hole Dia & (in) & 0.1895 & 0.1895 & 0.1895 & 0.1895 & 0.1895 & 0.1895 & 0.1895 & 0.1895 \\
\hline Crack Length@ Current Fastener & (in) & 0.000000 & 0.000000 & 0.000000 & 0.000000 & 0.000000 & 0.000000 & 0.000000 & 0.000000 \\
\hline Load.Magnit $\left(\mathrm{X}^{\wedge} 2+\mathrm{Y}^{\wedge} 2+\mathrm{Z}^{\wedge} 2\right)^{\wedge} 0.5$ & $(I b f)$ & 34.2 & 35.8 & 35.0 & 34.7 & 34.8 & 34.2 & 34.5 & 35.3 \\
\hline Ox Total Load & $(I b f)$ & -1.1 & -0.4 & -0.1 & 0.7 & 0.4 & 0.6 & 0.2 & -0.2 \\
\hline Oy Total Load & $(I b f)$ & 34.2 & 35.8 & 35.0 & 34.7 & 34.8 & 34.2 & 34.5 & 35.3 \\
\hline Oz Total Load & $(I b f)$ & 6.7 & 4.9 & 4.9 & 4.5 & 2.5 & 2.3 & 2.4 & 2.5 \\
\hline Angle & $(\mathrm{deg})$ & 91.82 & 90.62 & 90.21 & 88.80 & 89.32 & 89.08 & 89.66 & 90.36 \\
\hline
\end{tabular}



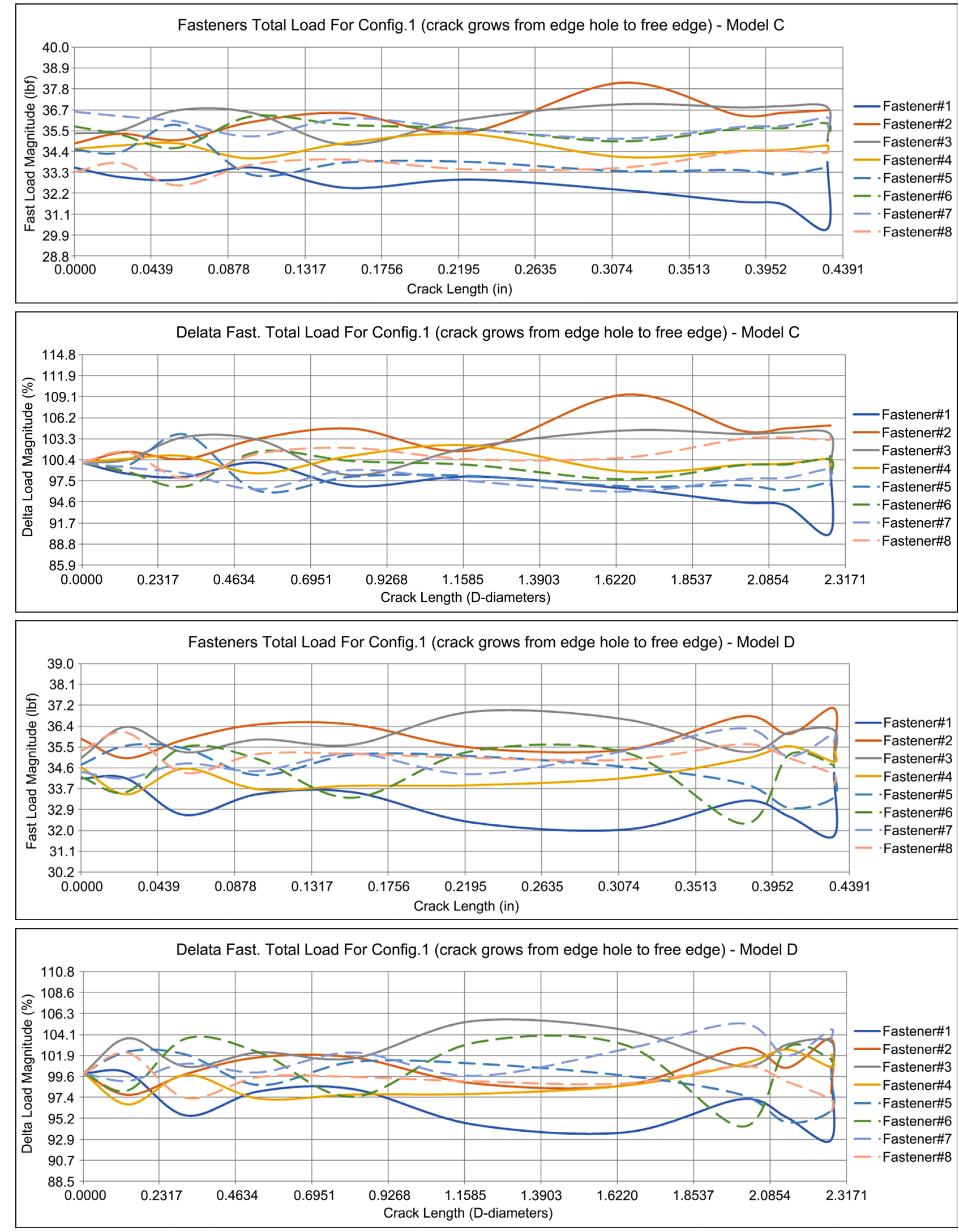

Figure 7. Variation of fastener loads in crack configuration 1 (crack growing from a hole to the free edge of the plate); (model C plates restrained in assembly, model D - simple laboratory testing configuration). 

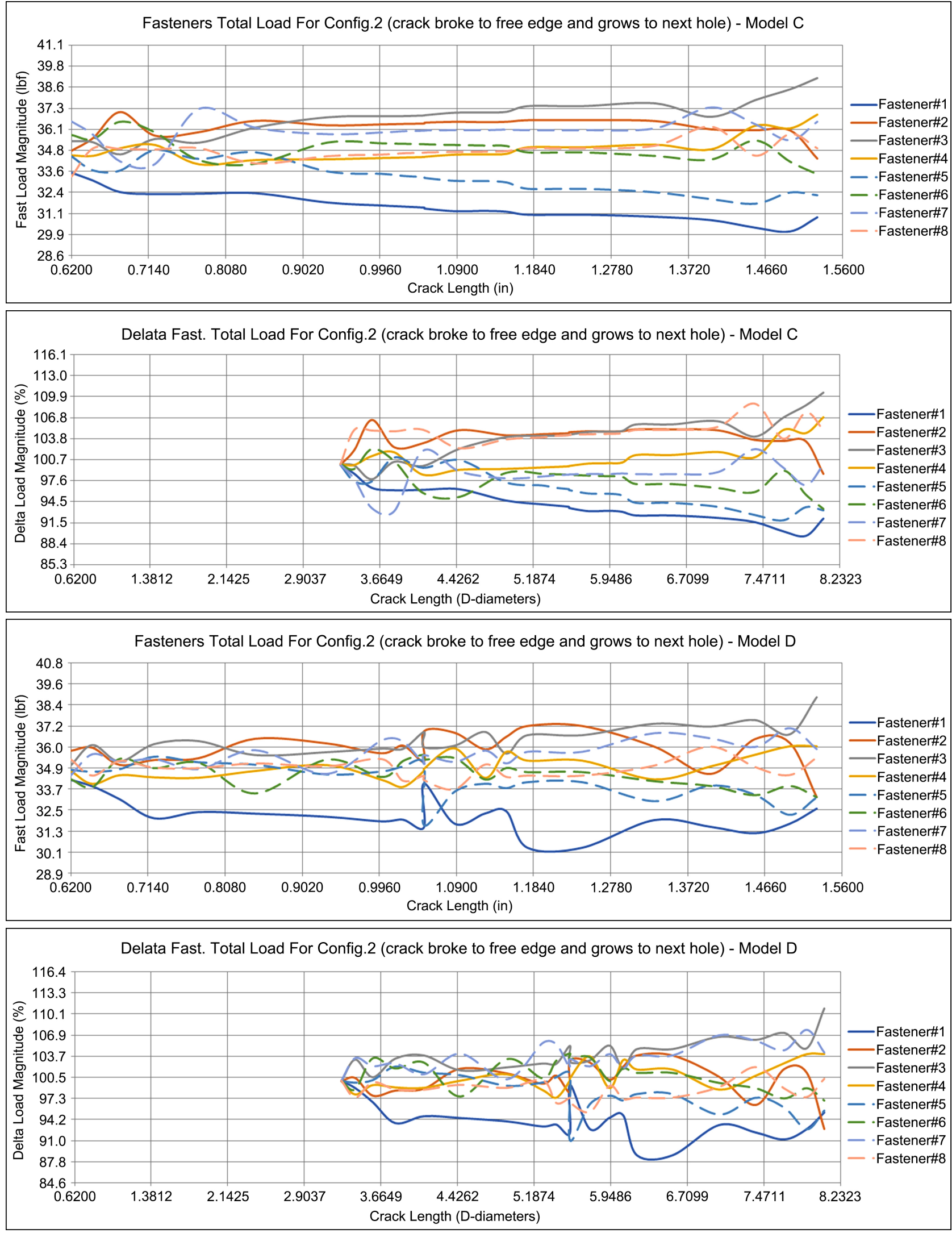

Figure 8. Variation of fastener loads in crack configuration 2 (after breaking the ligament a new crack growing from same hole to the next fastener hole); (model C - plates restrained in assembly, model D - simple laboratory testing configuration). 

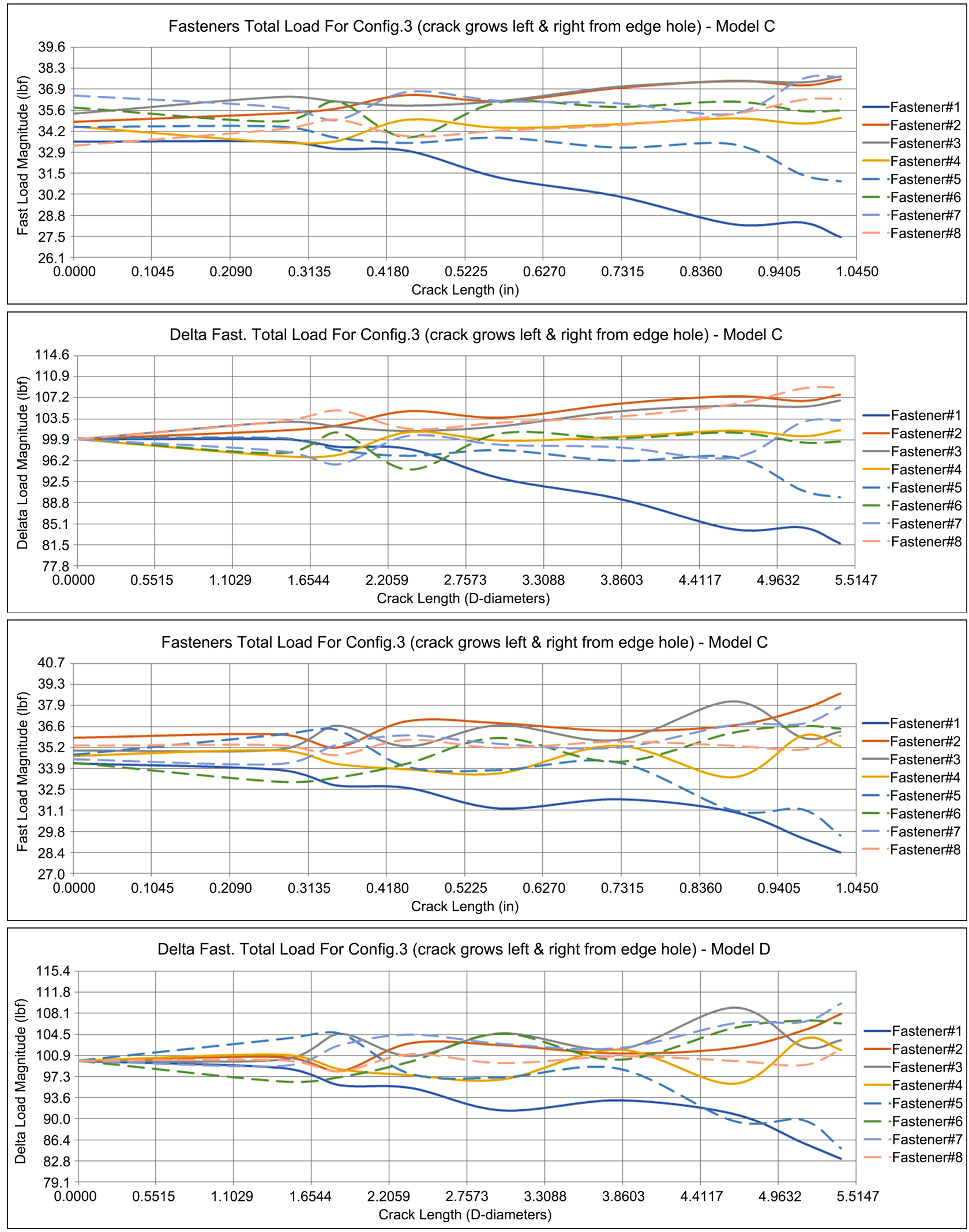

Figure 9. Variation of fastener loads in crack configuration 3 (two tips crack growing to the plate free edge and to the next fastener hole) (the diagrams are trimmed after this configuration becomes configuration 2); (model C - plates restrained in assembly, model D - simple laboratory testing configuration). 

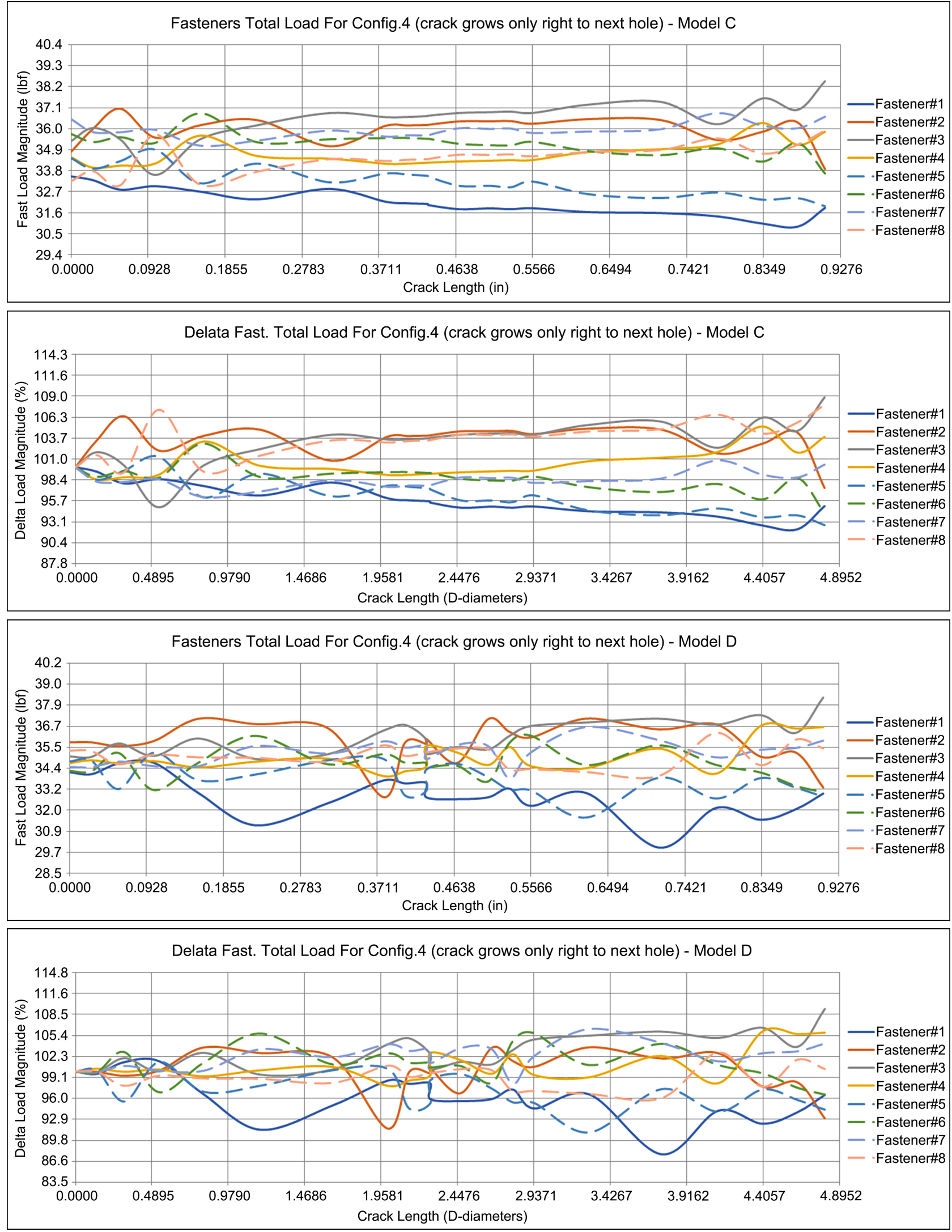

Figure 10. Variation of fastener loads in crack configuration 4 (one tip crack growing from internal hole \#2 to hole \#3); (model C - plates restrained in assembly, model D - simple laboratory testing configuration). 

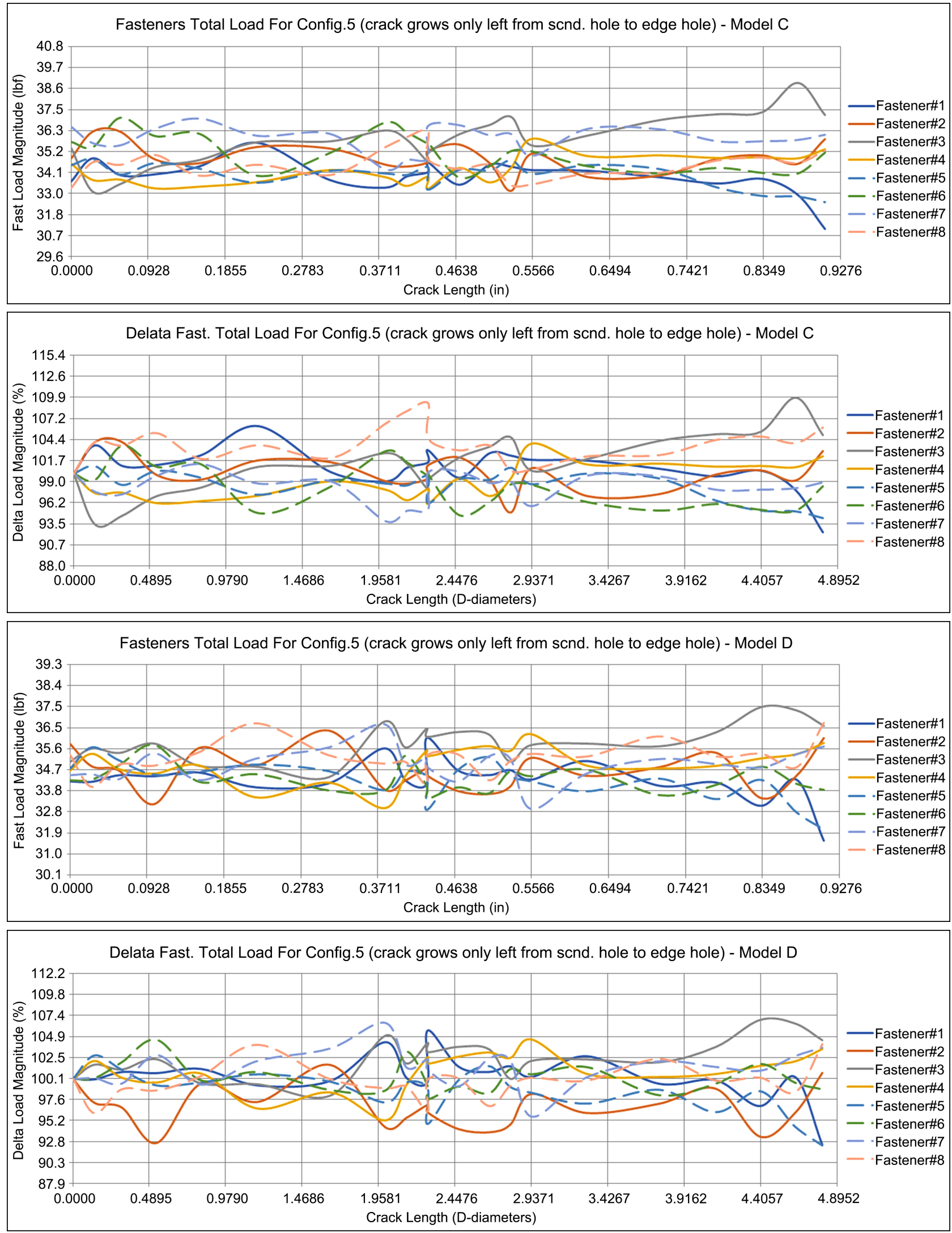

Figure 11. Variation of fastener loads in crack configuration 5 (one tip crack growing from internal hole \#3 to hole \#2); (model C - plates restrained in assembly, model D - simple laboratory testing configuration). 
For this reason the data reduction was somehow limited, with the results being post-processed only on a pin - by-pin basis for each crack configuration at 12 equidistant levels along the pin. Typical results for the Oy components of the bearing forces are shown in Figure 12 below. They are for two initial crack lengths, for the last increment before ligament fracture and after its fracture.

Because the plates were idealized using 2D elements located at the midplane of the plates and because, as shown in Figure 6, the faying surface between the top and bottom plates is between levels 4 and 5 of the pin, the bearing loads at the bottom of the hole in plate 1 needed to be multiplied by a factor of approx. 1.5. This was necessary in order to account for fact that the contact area in the plate is thinner than five pin levels ${ }^{7}$. A similar approach was applied for the distribution of the bearing loads in lower plate.

After the above correlation between the bearing loads in the pins and those at the hole surface, the results showed that:

- the bearing loads at the hole increase continuously from the fastener head (or from the collar) towards the separation surface. The maximum values are at the faying surface (after applying the factor for the transfer of the pin loads to the $2 \mathrm{D}$ plate) regardless of the cracked or uncracked condition. This aspect of the contact loads at pin-hole interfaces is described in more detail in publications like [7] [9] [16]. It is attributed in large portion to secondary bending and, to a lesser degree, to bolt tilting;

- the presence of the cracks at the hole or at full length have very little influence on the through thickness distribution of bearing loads (or stresses). At the faying surface they were less than $3 \%$ different from the baseline configuration, and followed the same pattern as the fastener shear load (including the "hook effect" after fracture),

- at the fastener hole next to the cracked one, because the total shear force increases with the crack length, the maximum bearing loads at the faying surface increase also by around $7 \%$ from their values in baseline configuration shown in Table 3,

- for the laboratory testing configuration, the distribution of the bearing loads was very similar, but the variations during the crack growth are negligible at both the cracked hole and at the adjacent one (less than $3 \%$ ).

The main drivers for both the small variations in the through thickness distribution of the bearing loads and for the difference in patterns of the two sets of resultsare the lateral restraints of the pates. A similar conclusion is presented in reference [7] where it is stated that "it was found that in cases of splice plates with un-symmetric reinforcement relative to the fastener holes, the secondary bending was significantly different on the two sides of the same fastener hole" and that the "approach must consider the distribution of contact forces between the plates and between the fastener heads and the plates (due to fastener tilt)"

${ }^{7}$ Simple analogies could be made with the weighting factor applied to an RBE3 when transferring a nodal concentrated force as a uniformly distributed load on the edge of a plate. 

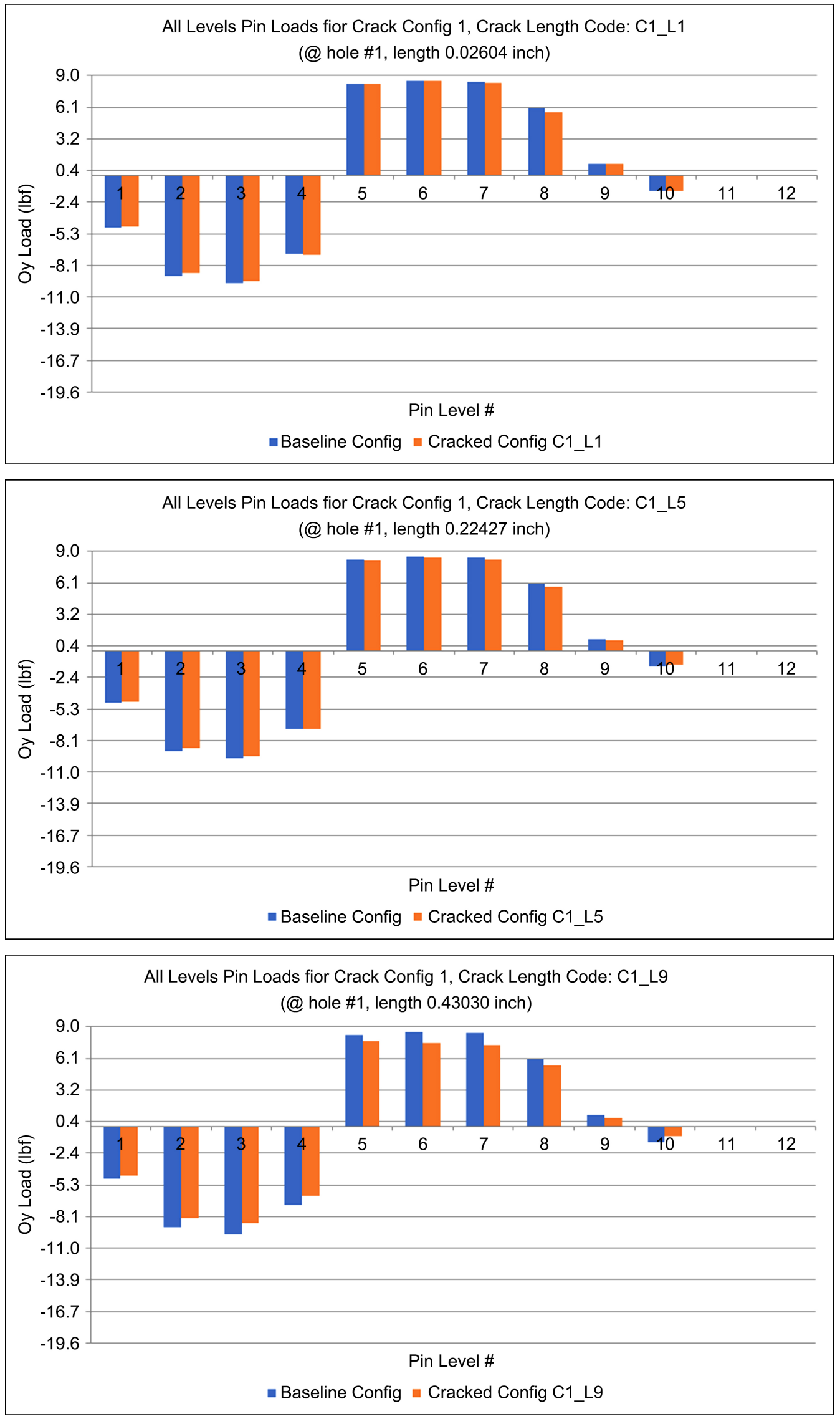


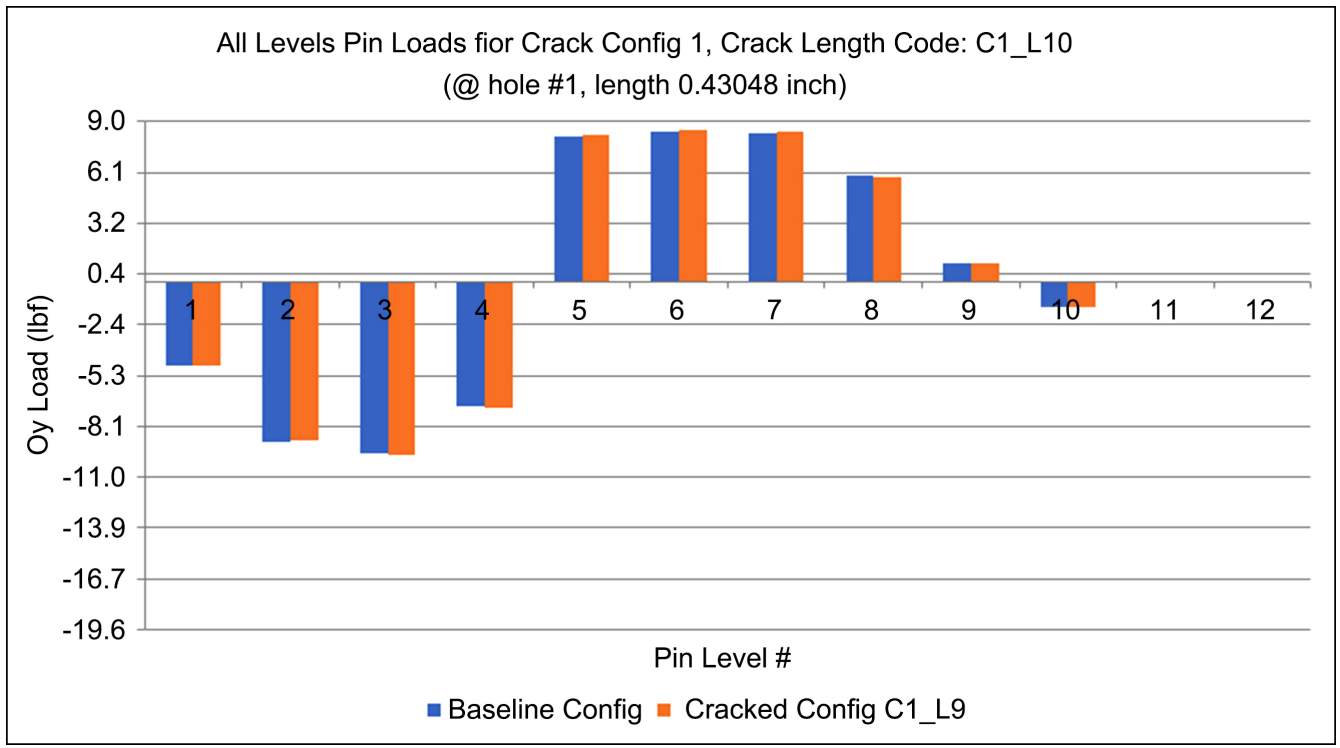

- Location of faying surface between the two plates (top plate P1 - left, bottom plate P2 - right) Layers 9 and 10 correspond to the pin-collar interfaces.

Figure 12. Distribution of bearing loads along the pin of fastener \#1.

From an engineering perspective, for real structures, the importance of the distribution in the bearing loads through the hole is the fact that for single shear joints or joints with large eccentricities, the cracks initiate in the thinnest part, at the hole edges located on the faying surface. This is even more relevant for the locations where single shear joints are inaccessible or for multi-layered joints with large eccentricities. For these types of details, the cracks cannot be easily detected without removing the bolt, and they can grow undetected to large lengths and promote the appearance of multi- site and/or multi-element fatigue damage in the structure.

Such cracks were found in several locations during the teardown stage after a full scale wing fatigue testing done in the last few years on a medium transport aircraft. Several cracks above one inch in length have been found in the rear spar webs, at the joints with the lower spar caps. The materials of web and spar caps was 7075 aluminium alloy, and at all the cracked locations discussed there were four or more layers stack ups with significant imbalance. In all these cases, the spar cap vertical flange and the web were sandwiched between thick fittings attached with two or three vertical rows of two large fasteners per row. The large fasteners were common to all the layers, and the local configurations can be described as hard points with high fastener load transfer. By the end of testing, the largest crack grew to several inches in the web, extended to several adjacent holes and caused significant cracking in the vertical flange of the spar cap. At all the other locations, the cracks have developed only in the webs remaining hidden until teardown.

Forensic examination conducted in Australia at Defence Science and Technology group (DST), established that the largest crack started in the web at a 
hole common to the web and spar flange and the fittings at around $6 \%$ of the testing program, and grew upwards towards the next hole. A secondary crack initiated from the opposite side of the same hole and grew towards the free edge at $25 \%$ of the program. The crack in the spar cap flange initiated at the same hole close to $50 \%$ of testing and grew both towards free edge and towards the spar cap horizontal flanges. The locations and the times for the initiation of these cracks were established using Quantitative Fractography and are shown in Figure 13.

The tested configuration was representative of production aircraft and of the assembly methods used, including the torque specifications for removable fasteners. At these locations there was very little if any secondary bending at the hole edges of the web, and the fact that cracks in the web were found at most locations of similar configuration indicates a high correlation with the distribution of bearing loads and with the influence of the clamping forces.

\subsection{Effect of Crack Length on the Far Field and Bypass Strip Loads and on the Running Loads}

Because for the single shear tension joint presented, the effects of secondary bending are significant, the results are split in two sub-groups:

1) The effects of the cracking configurations and of the crack lengths on the running loads in the membrane ${ }^{8}$,

2) The effects of secondary bending due to the load path eccentricity.

The results have been processed and they are shown in terms of internal loads for the convenience offered in visualization, coordinates transformations and in interpretations by the vectorial quantities.

1) The left side of Figure 14 shows the distribution of the membrane only running loads around the fastener holes and in the cartouche for the entire plate 1. On the right side the same distributions are shown just before the end of the crack growth in Config 3. With reference to the plots in this figure, the following two notes area made:

a) The plot on the right shows in the note at its lower right corner that the maximum shell force is $660 \mathrm{lbf} / \mathrm{in}$ (the location is at the crack tip near the free edge). The plate thickness is 0.063 " i.e. the tension stress in the ligament is 10.5 $k s i(72.4 \mathrm{MPa})$ which is well within the elastic range of the material and therefore the use of engineering linear elastic methods is appropriate;

b) Then the distribution of the internal loads, in the general vicinity of the fastener hole (i.e. within 10 - 15 hole diameters) is very dissimilar, and therefore the use of a common reference for the far-field or by-pass quantities, both in baseline and cracked configurations, was not considered adequate.

The results were post-processed as total internal forces carried by 0.435 inch (or 2.3D) wide strips symmetrical about each fastener axis and located at ap-

${ }^{8}$ Here the term membrane has the classical meaning in structural analysis and it denotes that part of the plate that reacts the externally applied loads in pure tension only (having a uniform stress in any $\mathrm{x}$-section perpendicular to the vector of the tension forces). 
proximately the same distance ahead or after the holes edges. Table 5 and Table 6 show that the strip approximation is adequate for the average running loads in baseline configuration because when the values are divided by the strip width, the result is very close to the uniformly distributed externally applied load (63 lbf/in).

When the crack grows, the running loads at each fastener are different from the externally applied load by variable amounts depending on the crack configuration and length. For single sided cracks at the first row of fasteners, they are within $\pm 20 \%$ of baseline values, and within $\pm 40 \%$ for the second row. For double sided cracks the variations are larger, in order of $\pm 50 \%$ at the crack location and at the immediately adjacent fasteners.

The support conditions for the lateral edges have a relatively small influence on the values of strip wide loads in the baseline configurations but, while the

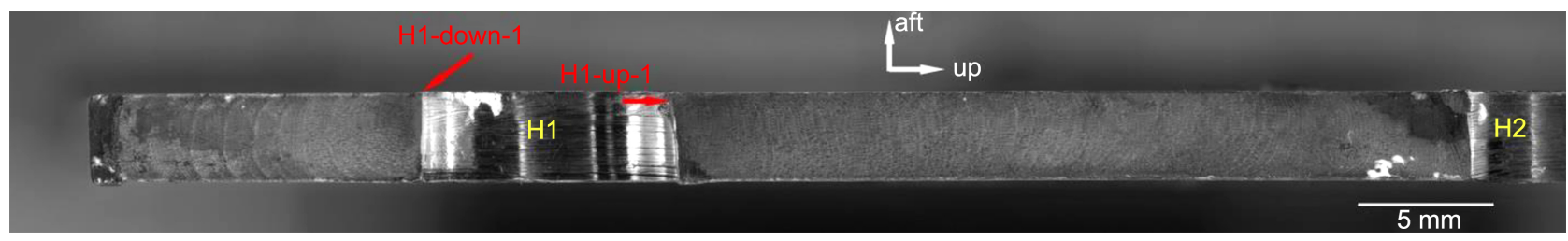

Figure 13. Stereoscopic image of the overall fracture surface for cracks in the webs of rear spar. (H1-down-1 and H1-up-1. The initial flaws are arrowed in red); (Reproduced with the permission of DST).

Table 5. Baseline total far field loads on a $0.455 "$ wide strip ahead of the fasteners for model C.

\begin{tabular}{|c|c|c|c|c|c|c|c|c|c|}
\hline \multicolumn{10}{|c|}{ Baseline FarField Forces At All Fasteners (summed over 2.3D, symmetrical about fast C/L) } \\
\hline Model & $\mathrm{C}$ & Pin/Hole & Pin/Hole & Pin/Hole & Pin/Hole & Pin/Hole & Pin/Hole & Pin/Hole & Pin/Hole \\
\hline Panel & $\mathrm{P} 1$ & 1 & 2 & 3 & 4 & 5 & 6 & 7 & 8 \\
\hline Hole Dia & (in) & 0.1895 & 0.1895 & 0.1895 & 0.1895 & 0.1895 & 0.1895 & 0.1895 & 0.1895 \\
\hline Crack Length@ Current Fastener & (in) & 0.000000 & 0.000000 & 0.000000 & 0.000000 & 0.000000 & 0.000000 & 0.000000 & 0.000000 \\
\hline Lateral (Ox) Total Load & $(I b f)$ & 7.856 & 7.867 & 7.880 & 7.931 & 3.891 & 3.993 & 3.995 & 4.003 \\
\hline Axial (Oy) Total Load & $(I b f)$ & 26.901 & 26.904 & 26.958 & 26.980 & 10.509 & 10.233 & 10.191 & 10.043 \\
\hline Average Shear Flow & $(I b f l i n)$ & -0.099 & 0.004 & -0.021 & 0.093 & -0.191 & 0.053 & -0.055 & 0.216 \\
\hline
\end{tabular}

Table 6. Baseline total far field loads on a $0.455 "$ wide strip ahead of the fasteners for model D.

\begin{tabular}{|c|c|c|c|c|c|c|c|c|c|}
\hline \multicolumn{10}{|c|}{ Baseline FarField Forces At All Fasteners (summed over 2.3D, symmetrical about fast C/L) } \\
\hline Model & $\mathrm{D}$ & Pin/Hole & Pin/Hole & Pin/Hole & Pin/Hole & Pin/Hole & Pin/Hole & Pin/Hole & Pin/Hole \\
\hline Panel & $\mathrm{P} 1$ & 1 & 2 & 3 & 4 & 5 & 6 & 7 & 8 \\
\hline Hole Dia & (in) & 0.1895 & 0.1895 & 0.1895 & 0.1895 & 0.1895 & 0.1895 & 0.1895 & 0.1895 \\
\hline Crack Length@Current Fastener & (in) & 0.000000 & 0.000000 & 0.000000 & 0.000000 & 0.000000 & 0.000000 & 0.000000 & 0.000000 \\
\hline Lateral (Ox) Total Load & $(1 b f)$ & -0.114 & 0.418 & 0.440 & -0.137 & 0.730 & 0.480 & 0.444 & 0.727 \\
\hline Axial (Oy) Total Load & $(I b f)$ & 26.013 & 27.504 & 27.468 & 26.101 & 9.282 & 9.848 & 10.107 & 9.261 \\
\hline Average Shear Flow & $($ lbflin) & 0.321 & -0.040 & 0.038 & -0.317 & -0.219 & -0.235 & 0.366 & 0.310 \\
\hline
\end{tabular}


Patran 2019 08-May-20 14:47:41

Fringe: SC1:Step 4:CRACK FROM RHS EDGE OF H1 GROWS TOWARDS H2, A1:Non-linear: 400.\% of Load, Shell Forces, Force Resultant, Y Comp

Deform: SC1:Step 4:CRACK FROM RHS EDGE OF H1 GROWS TOWARDS H2, A1:Non-linear: 400.\% of Load, Displacement, Translational,, (NON

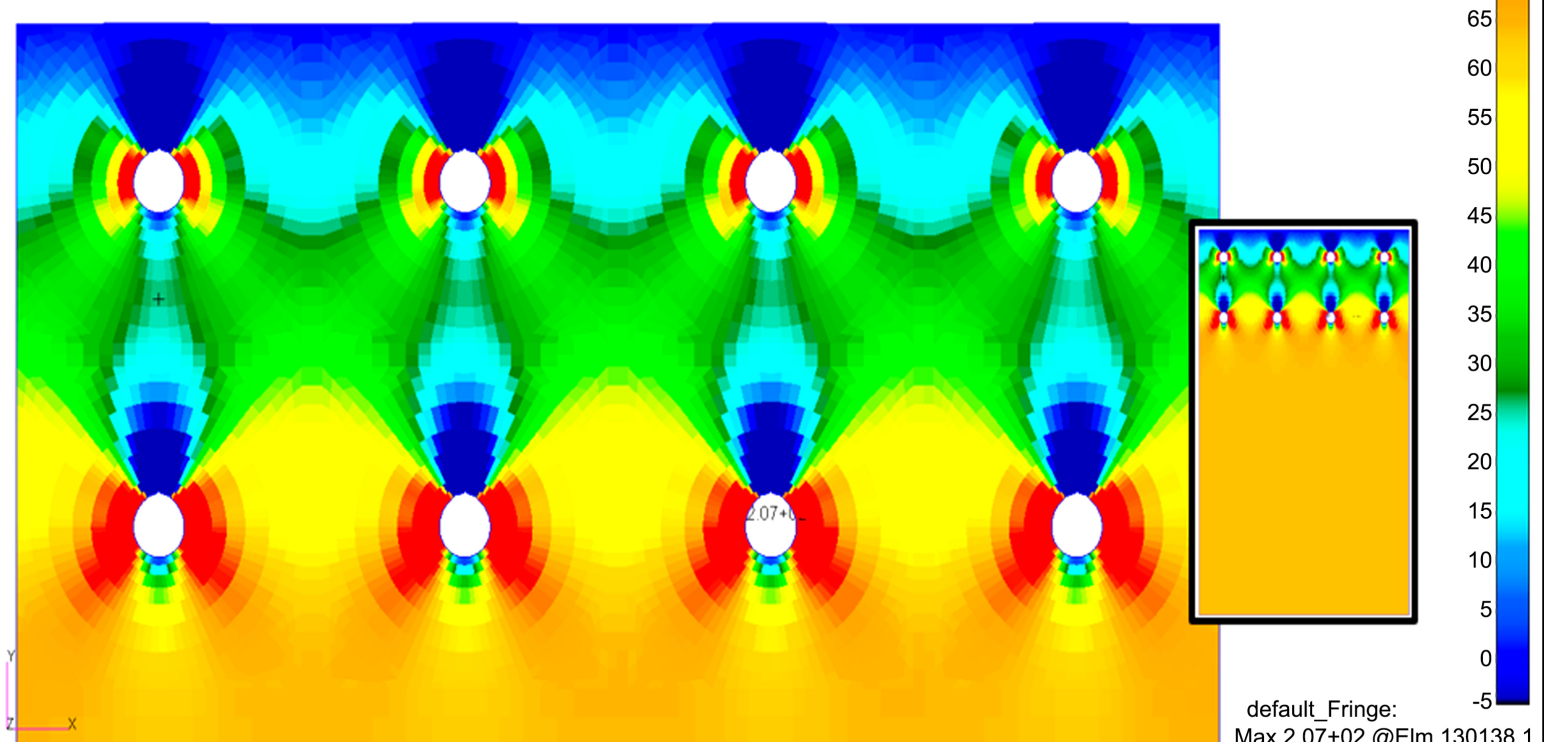

$\operatorname{Max} 2.0 \overline{7}+02 @ E I m 130138.1$ Min-8.06+02@Elm 150120.1 default_Deformation: Max 3.06-02 @Nd 197639

Patran 2019 08-May-20 14:29:52

Fringe: SC1:Step 4:CRACK GROWS FROM H1 TOWARDS FRRE EDGE, A1:Non-linear: 400.\% of Load, Shell Forces, Force Resultant, Y Component Deform: SC1:Step 4:CRACK GROWS FROM H1 TOWARDS FRRE EDGE, A1:Non-linear: 400.\% of Load, Displacements, Translational,, (NON-LAYER

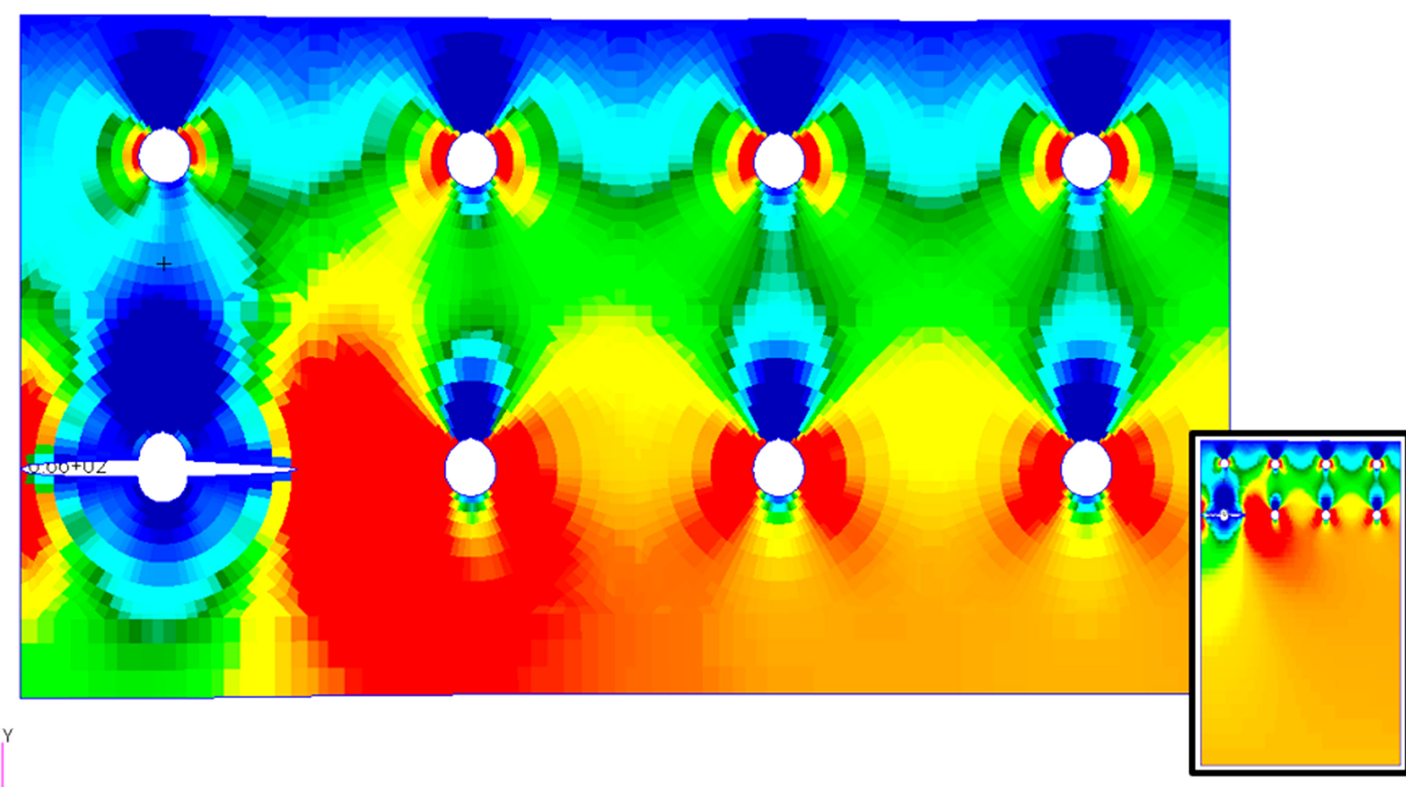

default_Fringe:

Max 6.66+02@EIm 110941.1 Min -8.76+02@Elm 170121.1

default Deformation: Max 3.17-02 @Nd 197552

Figure 14. Plot of shell in element Oy forces in baseline configuration and at the end of Config 3 for model C. (Fringe range: -5 lbf/in to 75 lbf/in; Coordinate Frame: CID 0; Averaging: Domain - none ; Method - average/derive; Extrapolation: -centroid); (Deformations: true scale; Scale Factor 200:1). 
cracks grow or after the fracture of the ligament(s), the patterns of variations at individual fastener locations are different. The evolution of the far field loads (in strip wide basis) for all crack configurations analysed are shown in Figure 15 and Figure 16. The curves have been plotted in real force and distance units for clarity and to overcome the "change sign" alerts occurring when building envelope summaries of percent variations from baseline configuration.

All curves are relatively smooth and can be easily represented as formulas (polynomial, splines etc.). Conversion to running loads or average stresses involves only divisions by specific constants dependent on hole location relative to the crack and/or the reference value preferred (strip wide or gross average far-field). For non-dimensional or parametric form, a few more runs with different plate gauges and fastener sizes are required.

For by-pass load the curves are not shown because for the first row the bypass curves are almost identical to the far-field curves in Figure 15 and Figure 16, and the bypass values for the second row are almost zero due to the vicinity of plate's end. It is noted that after the cracked hole, there is significant variation in the strip-wide bypass membrane force.

2) The distribution of moments due to secondary bending at the configurations and crack lengths exemplified are shown in Figure 17. The strip-wide approach detailed before provides the averages of the far-field and bypass bending moment ahead and after each fastener hole. All the fastener level variation diagrams are quite flat in all configurations and therefore they are not included in the results shown.

With the two sets of average internal loads within the band, the components in Oy direction for the far-field and bypass stresses can be easily determined at any plate depth using the classical structural analysis formulas.

The variations functions for shear flows follow similar patterns, but the values are small and therefore, the curves are not shown in this presentation.

\subsection{Effect of Crack Length on the Far Field and Bypass Stresses at Outer Surfaces of the Plates}

Because a large proportion of testing is based on the strains collected by strain gauges mounted on the outer surfaces of the parts, the results this section present the approach and the results for the far-field and bypass stresses on the surfaces. The added complexities in processing tensorial quantities were minimized but not quite removed by using only planes parallel with the reference coordinate system (CID 0) for the classical engineering formulations or by using the 2D min-max. invariant formulations (and principal angles) of the stresses.

The post processing was done only for the outer surfaces of the plate $1\left(0.063^{\prime \prime}\right.$ thick) and are shown for the same configurations exemplified in the running loads formulations, i.e. the averaging used was also the strip wide approach to reduce the variability in the stress fields, and suggests a pragmatic approach for quantification and for calibrations during laboratory testing. 

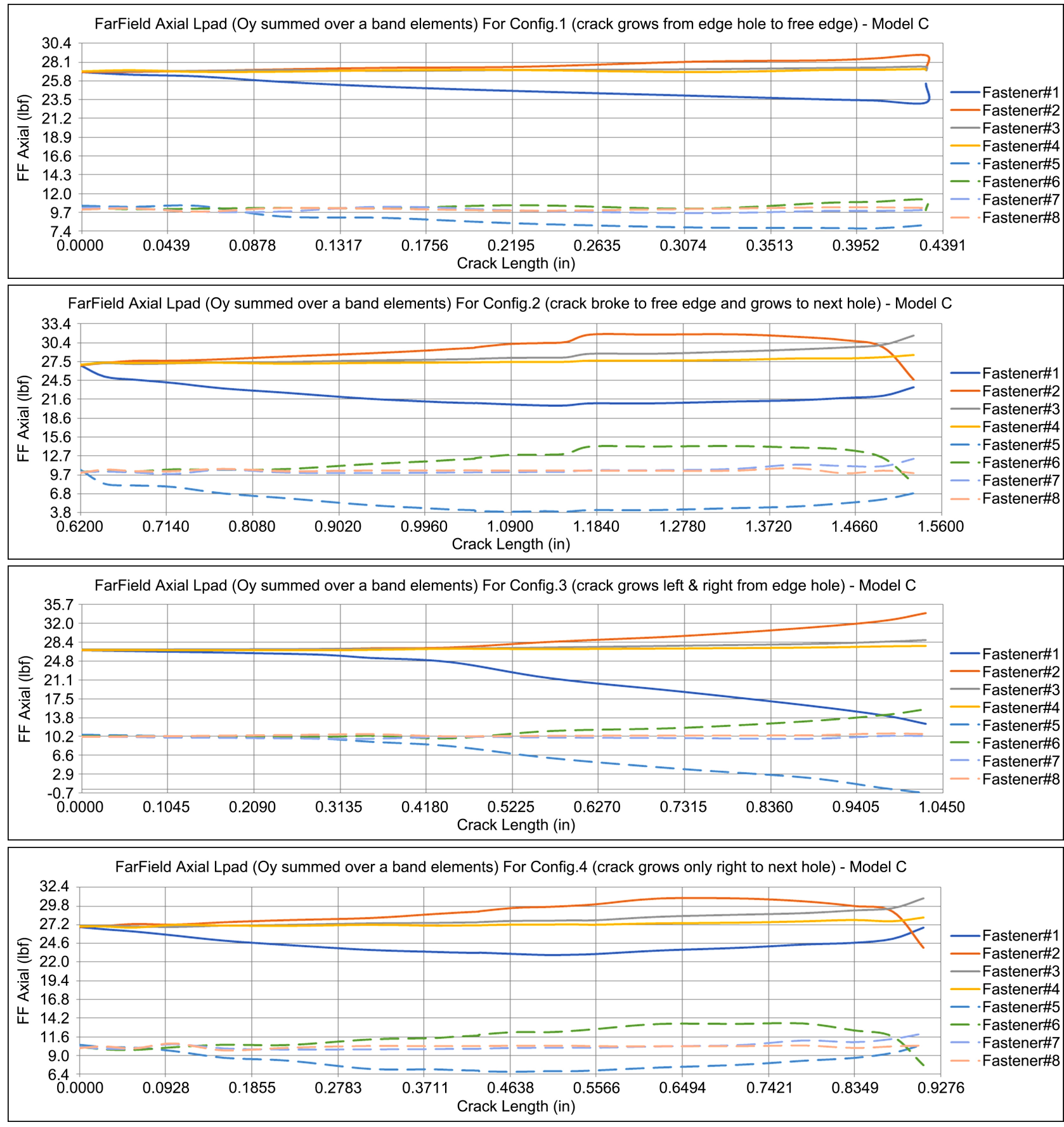

FarField Axial Lpad (Oy summed over a band elements) For Config.5 (crack grows only left from scnd. hole to edge hole) - Model C

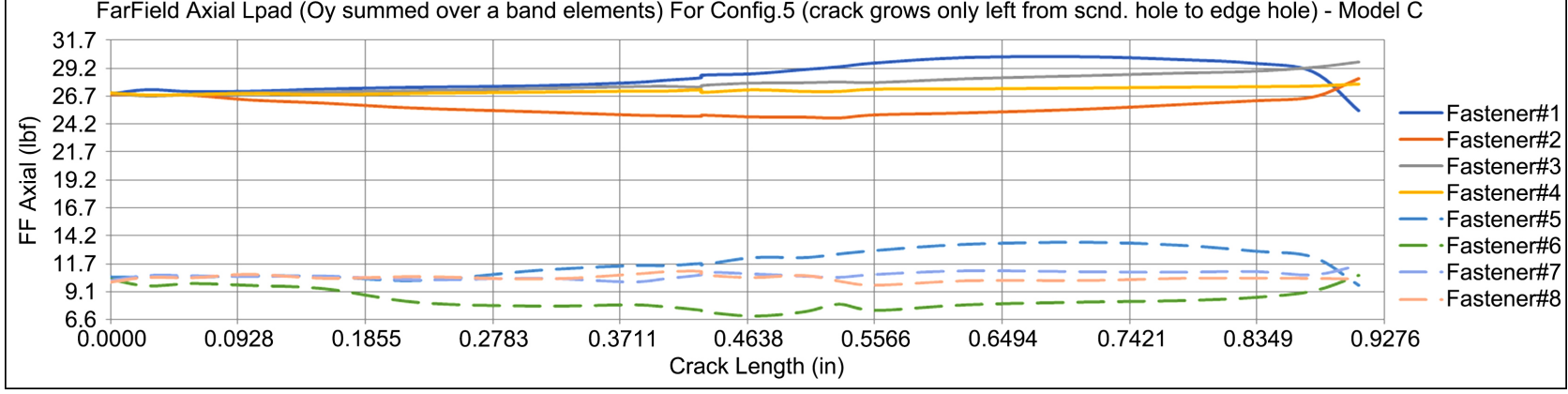

Figure 15. Distribution of strip wide far field tension forces during crack growth of Model C. 

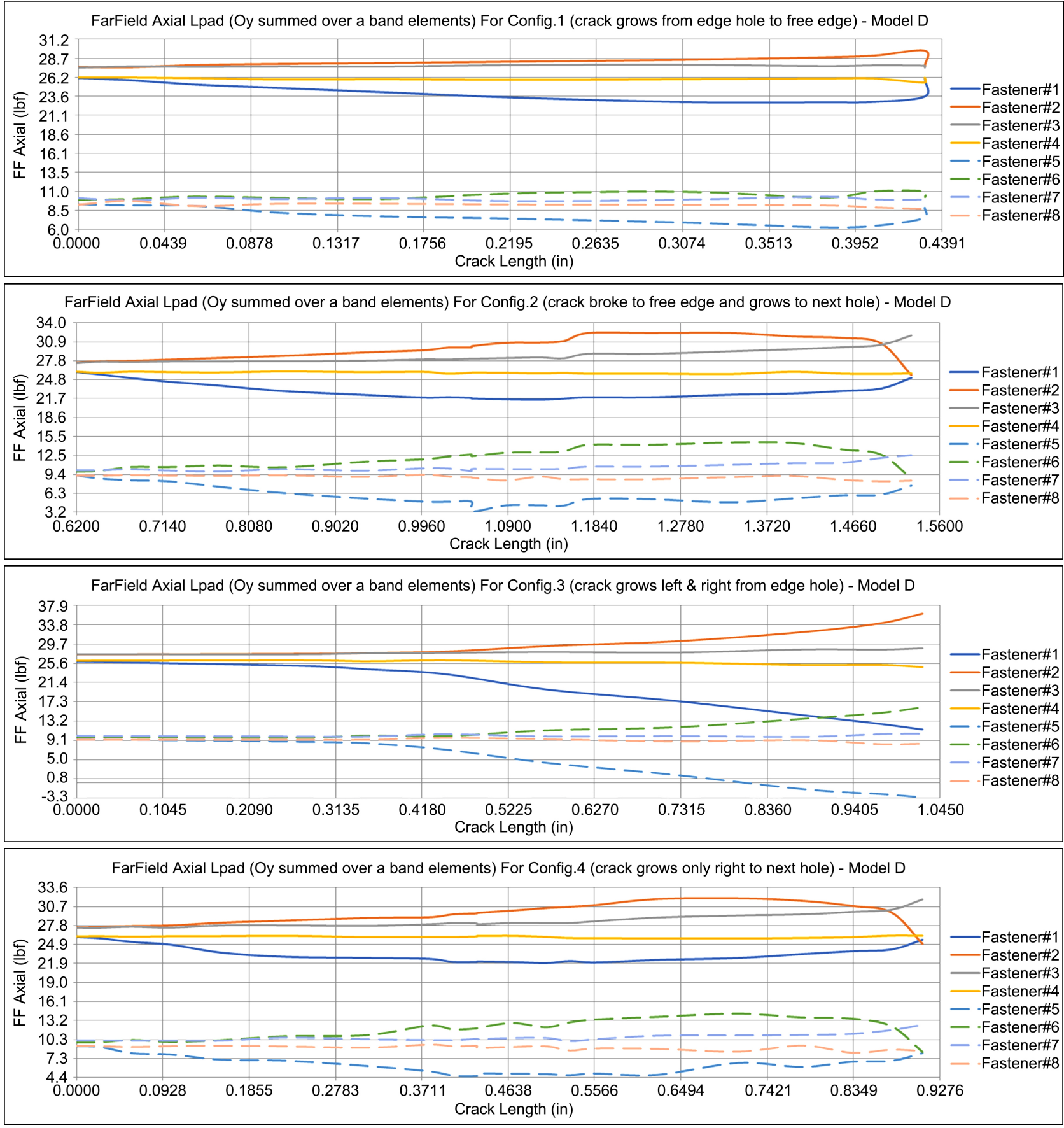

FarField Axial Lpad (Oy summed over a band elements) For Config.5 (crack grows only left from scnd. hole to edge hole) - Model D

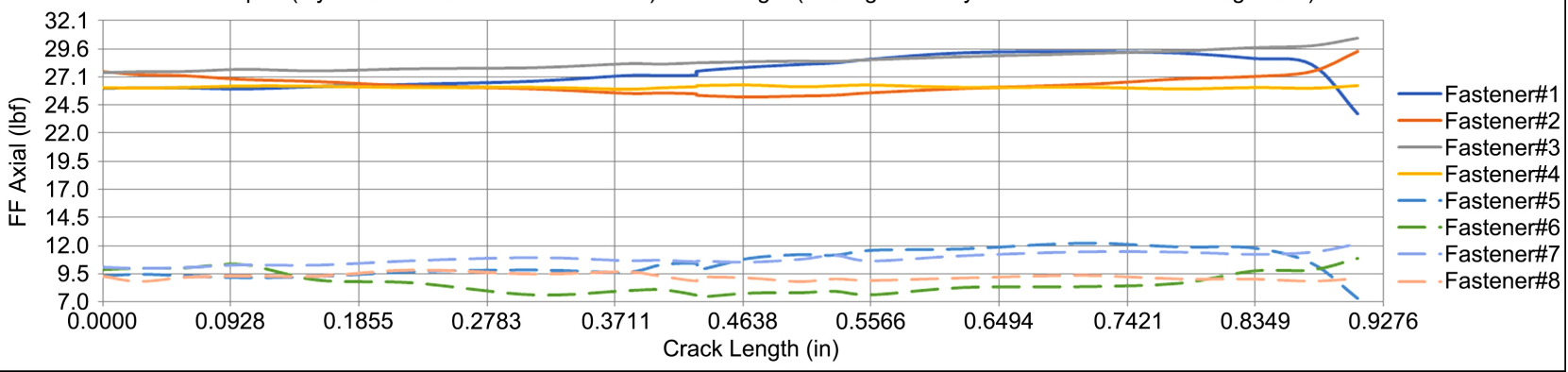

Figure 16. Distribution of strip wide far field tension forces during crack growth of Model D. 
Fringe: SC1:Step 4:CRACK FROM RHS EDGE OF H1 GROWS TOWARDS H2, A1:Non-linear: 400.\% of Load, Shell Forces, Moment Resultant, X Com Deform: SC1:Step 4:CRACK FROM RHS EDGE OF H1 GROWS TOWARDS H2, A1:Non-linear: 400.\% of Load, Displacements, Translational,, (NON
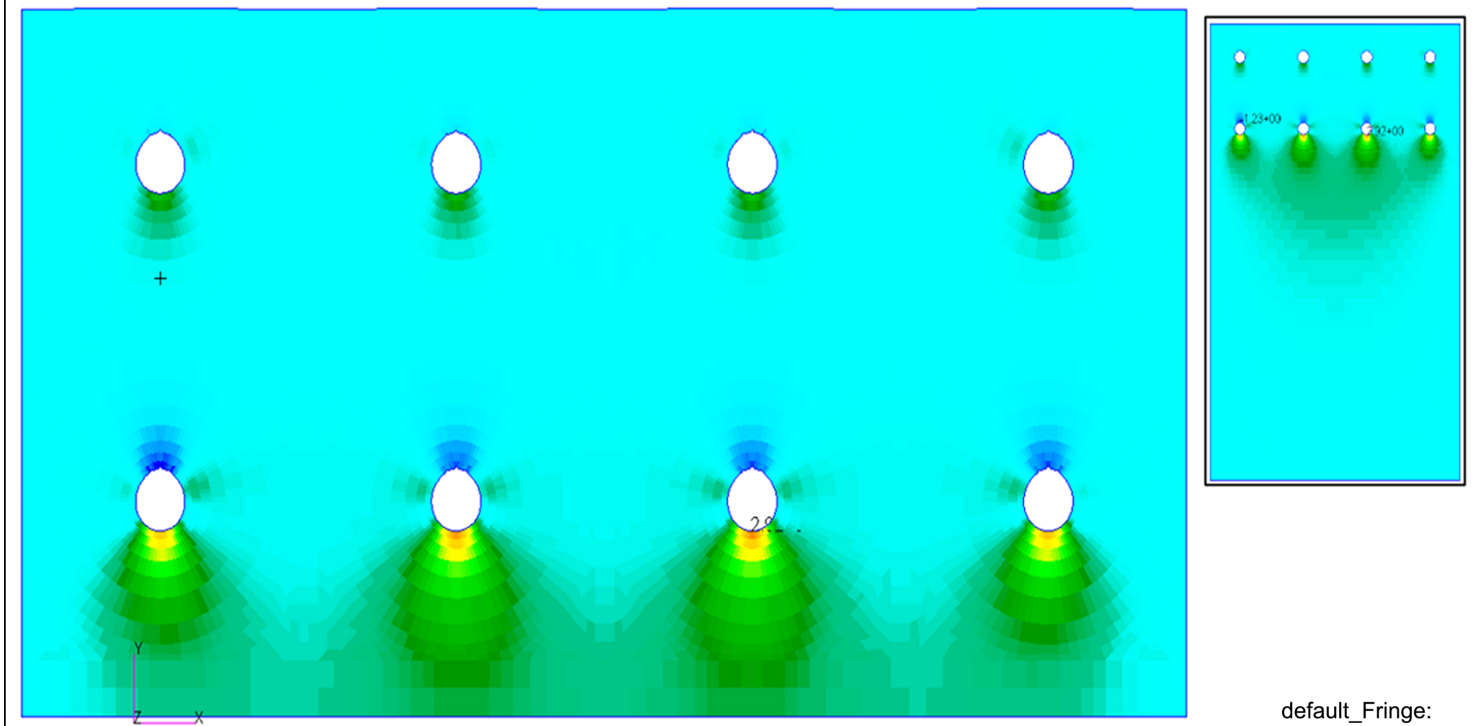

default_Fringe:

Fringe: SC1:Step 4:CRACK GROW FROM H1 TOWARDS FRRE EDGE, A1:Non-linear: 400.\% of Load, Shell Forces, Moment Resultant, X Componen Deform: SC1:Step 4:CRACK GROW FROM H1 TOWARDS FRRE EDGE, A1:Non-linear: 400.\% of Load, Displacements, Translational,, (NON-LAYER

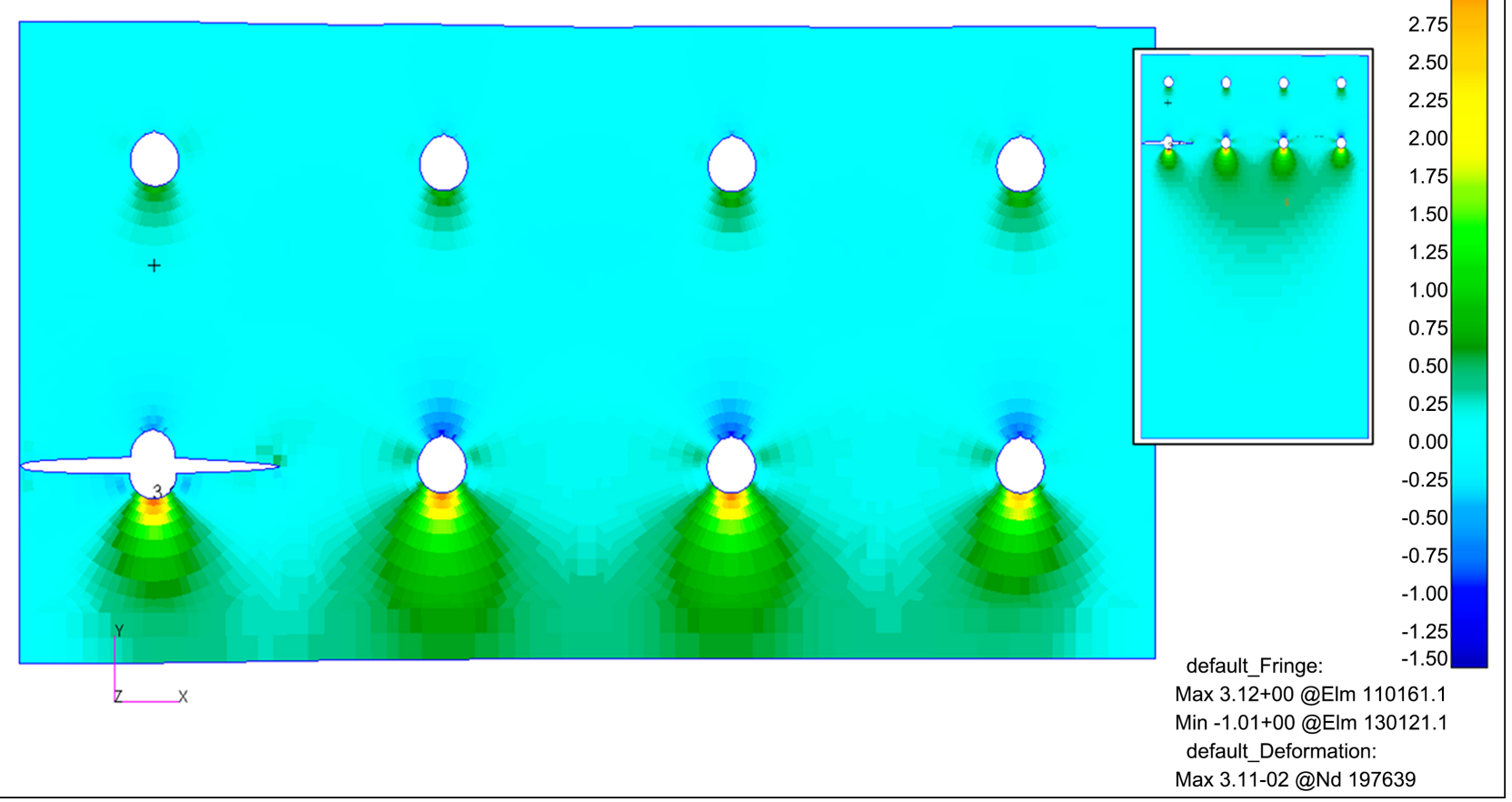

Figure 17. Plot of shell Mx moments due to secondary bending in baseline configuration and at the end of Config 3 for model C. (Fringe range: $-1.5 \mathrm{lbf}^{*}$ in/in to $3.5 \mathrm{lbf}^{*}$ in/in; Coordinate Frame: CID 0; Averaging: Domain - none; Method - average/derive; Extrapolation: - centroid); (Deformations: true scale; Scale Factor 200:1). 
Secondary bending effects are included in the results presented, but friction and fastener pretension effect are not accounted for. For laboratory testing lubrication between the faying surfaces might be needed to eliminate friction load transfers and match the analysis assumptions.

For the direct stresses $\sigma_{x}$, and $\sigma_{y}$ (strictly in CID0) "average" values were constructed by using virtual equivalent forces developed in a strip of unitary thickness that were divided by the cross sectional area of the strip. For the shear stresses $\tau_{\mathrm{xy}}$ (in CID 0), the min-max principal stresses ${ }^{9}$ and the maximum shear stresses, the "average" value was taken as the statistical median value of the these stresses for the 10 elements in each strip. This is because in the early post processing it was found that sometimes:

- the differences in the principal angles of any two elements were too big for, at least a rational, if not entirely rigorous, basis of averaging;

- the differences between the maximum and the minimum element values were disproportionately large in comparison with the values for each element.

An example of baseline values and outcomes of preliminary checks for the baseline stresses at the inner surface (Z1) in model C are shown in Table 7 and at the outer surface (Z2) in Table 8.

For the baseline configuration, the results of "averagings" are quite good for $\sigma_{y}$ (marked as Fy in the tables). A quick averaging between the far-field values stresses on inner and outer surface at each hole in the first row gives around $1000 \mathrm{psi}$, same as the tension stress of the external load, for both configurations. It was found that the there are no practical differences in these values for the two types of plate lateral restraints.

The effects of secondary bending are though substantial as illustrated also in Figure 18. At the outer surfaces the $\sigma_{y}$ components of the secondary bending stresses, are 2.5 times higher than the membrane tension stresses, resulting in compressive stresses for most of the upper surface. The secondary bending effects are however relatively easy to estimate from the strain gauges data on the free face once the secondary bending is taken into consideration. To remove the need of lubrication between the faying surfaces, friction data needs to be included in model D.

Results of a very similar nature were found for the other crack configurations. However, the variations of the surface axial stresses as functions of crack length weregenerally different for each configuration and fastener location both at the inner and outer surfaces. The secondary bending makes the influence of the cracks to be less noticeable than on the curves for fastener loads or the membrane running forces. The diagrams in Figure 19 to Figure 23 illustrate the above results for the far field stress.

${ }^{9}$ Calculated inside MSC Patranv19 end extracted as results for post-processing. Small corrections were done, where necessary, for the min principal values, as in this version, the outputs for the $2 \mathrm{D}$ min principal stress were truncated to zero when the max principal was large. Similar for max principal values, when the min principal was large. The principal angles were all correct when checked using the stress values in CID 0 . 
Patran 2019 10-May-20 11:34:52

Fringe: SC1:Step 4:CRACK FROM RHS EDGE OF H1 GROWS TOWARDS H2, A1:Non-linear: 400.\% of Load, Stress Tensor.. Y Component, At Z1 Deform: SC1:Step 4:CRACK FROM RHS EDGE OF H1 GROWS TOWARDS H2, A1:Non-linear: 400.\% of Load, Displacements, Translational,, (NON

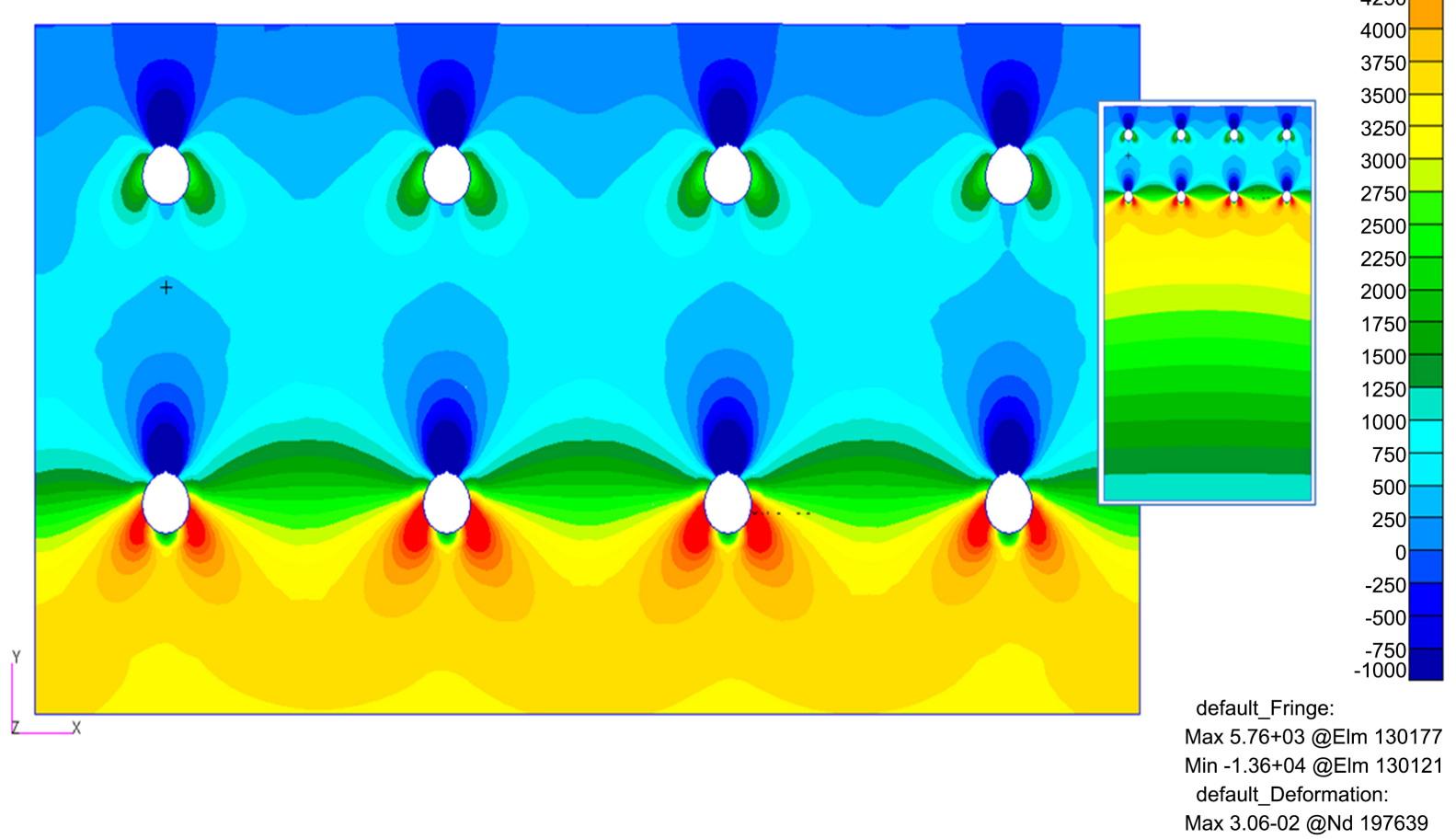

Patran 2019 10-May-20 11:22:43

Fringe: SC1:Step 4:CRACK GROWS FROM H1 TOWARDS FRRE EDGE, A1:Non-linear: 400.\% of Load, Stress Tensor.. Y Component, At Z1

Deform: SC1:Step 4:CRACK GROWS FROM H1 TOWARDS FRRE EDGE, A1:Non-linear: 400.\% of Load, Displacements, Translational,, (NON-LAYER

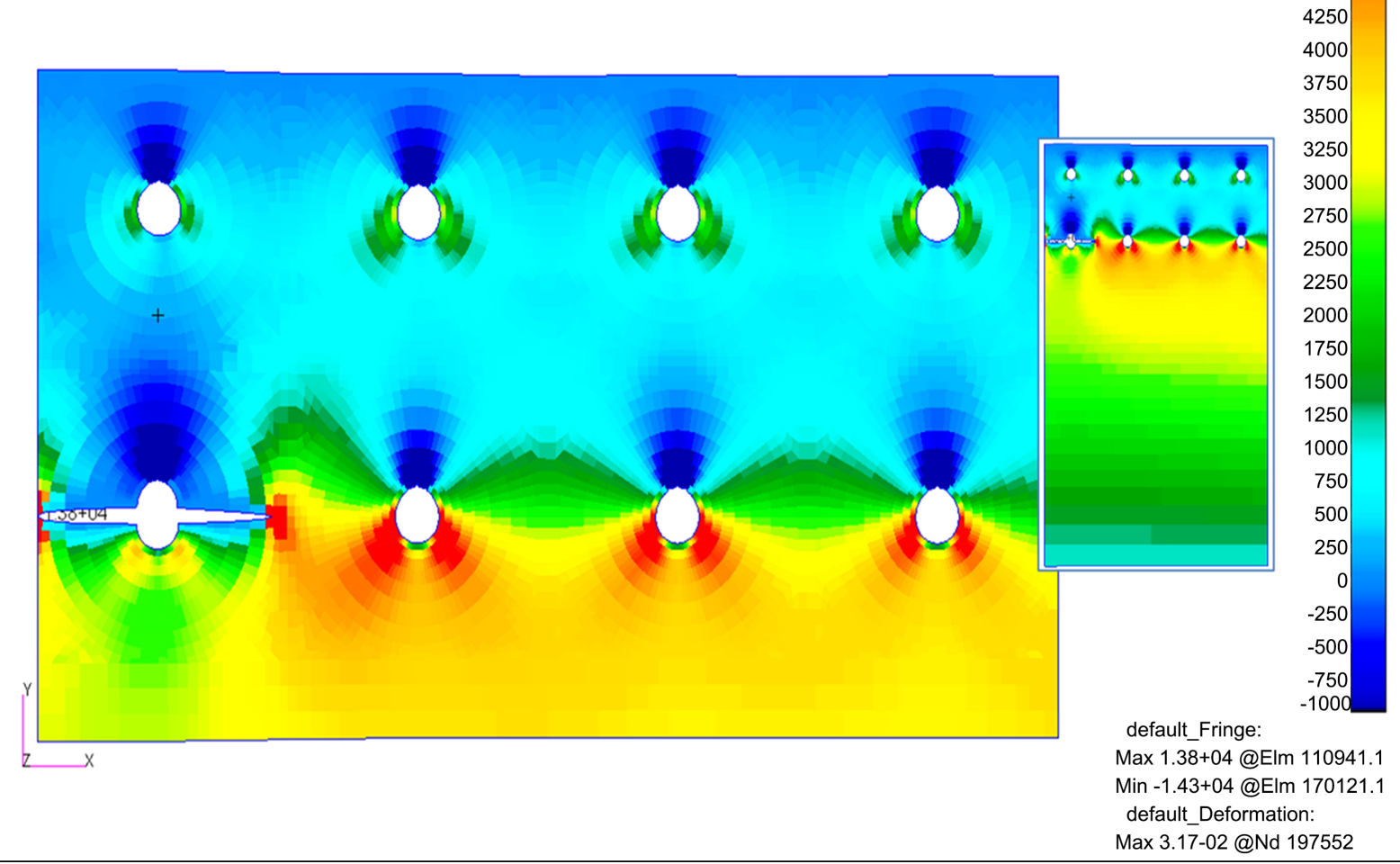


Patran 2019 10-May-20 11:32:42

Fringe: SC1:Step 4:CRACK FROM RHS EDGE OF H1 GROWS TOWARDS H2, A1:Non-linear: 400.\% of Load, Stress Tensor.. Y Component, At Z2 Deform: SC1:Step 4:CRACK FROM RHS EDGE OF H1 GROWS TOWARDS H2, A1:Non-linear: 400.\% of Load, Displacements, Translational,, (NON

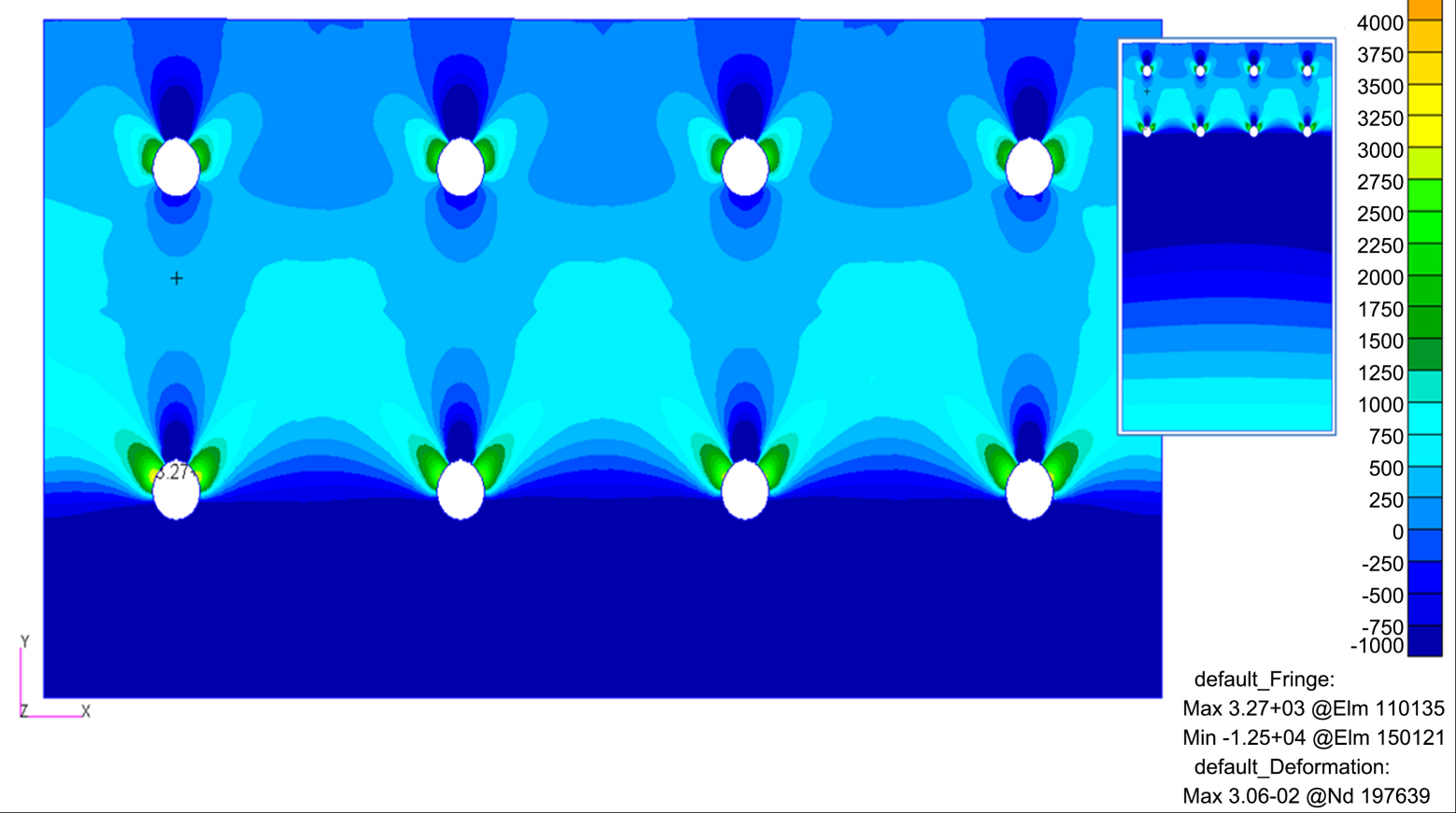

Patran 2019 10-May-20 11:19:39

Fringe: SC1:Step 4:CRACK GROWS FROM H1 TOWARDS FRRE EDGE, A1:Non-linear: 400.\% of Load, Stress Tensor.. Y Component, At Z2

Deform: SC1:Step 4:CRACK GROWS FROM H1 TOWARDS FRRE EDGE, A1:Non-linear: 400.\% of Load, Displacements, Translational,, (NON-LAYER

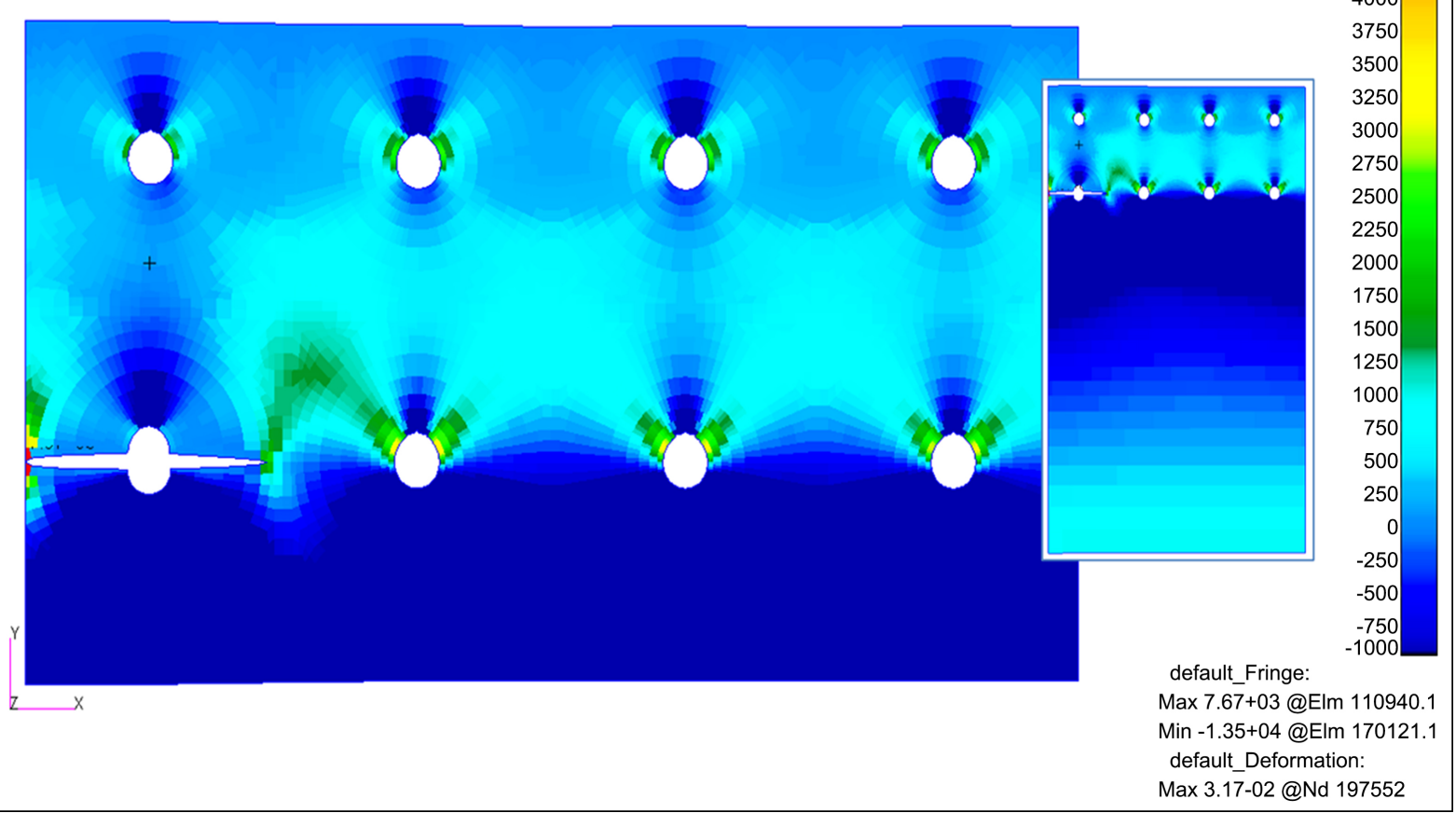

Figure 18. Plot of the surface stress components in Oy in baseline configuration and at the end of Config 3 for model C. (Fringe range: -1500 psi to 3500 psi; Coordinate Frame: CID 0; Averaging: Domain - none; Method - average/derive;Extrapolation: - centroid); (Deformations: true scale; Scale Factor 200:1). 

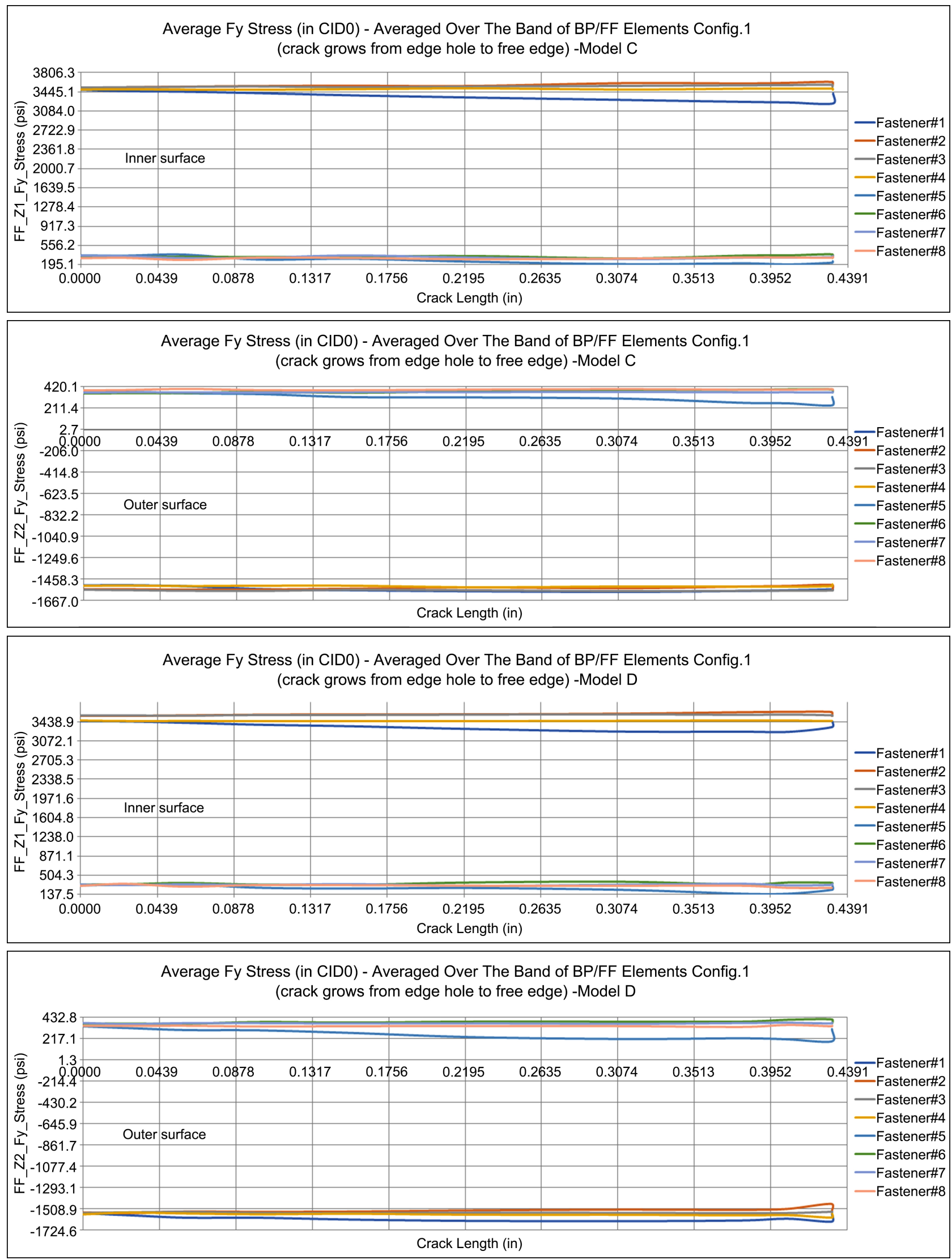

Figure 19. Distribution of strip wide averages of far-field stress on upper and lower surfaces as function of crack length in Config. 1. 


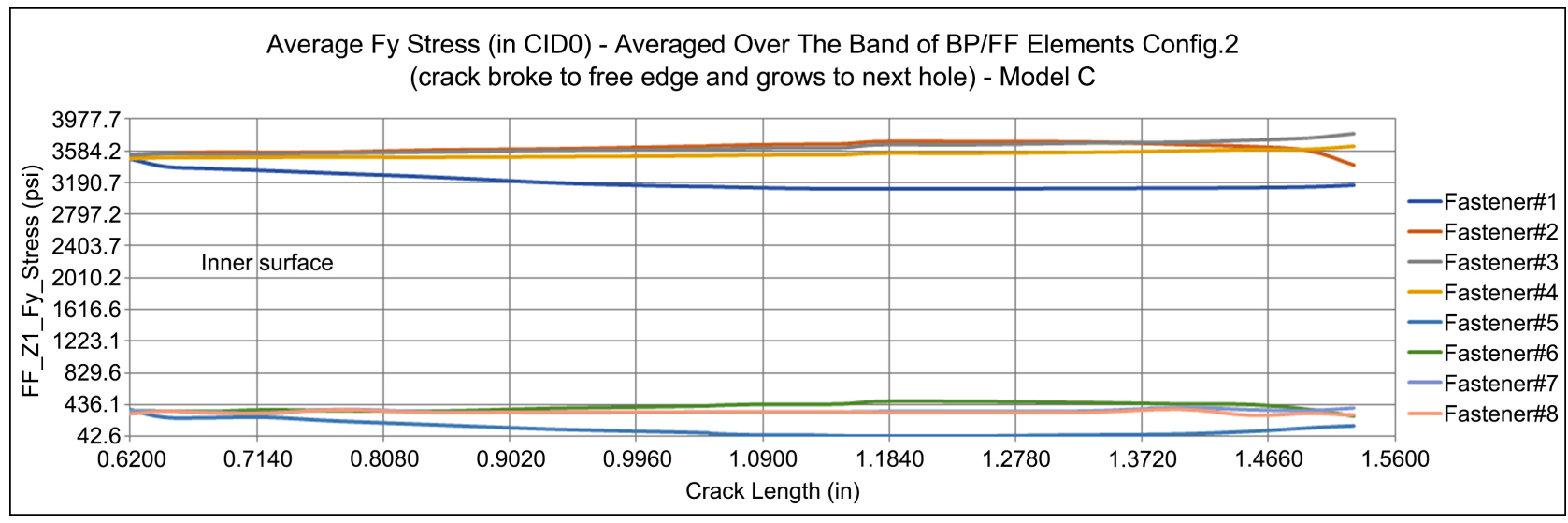

Average Fy Stress (in CIDO) - Averaged Over The Band of BP/FF Elements Config.2 (crack broke to free edge and grows to next hole) - Model C
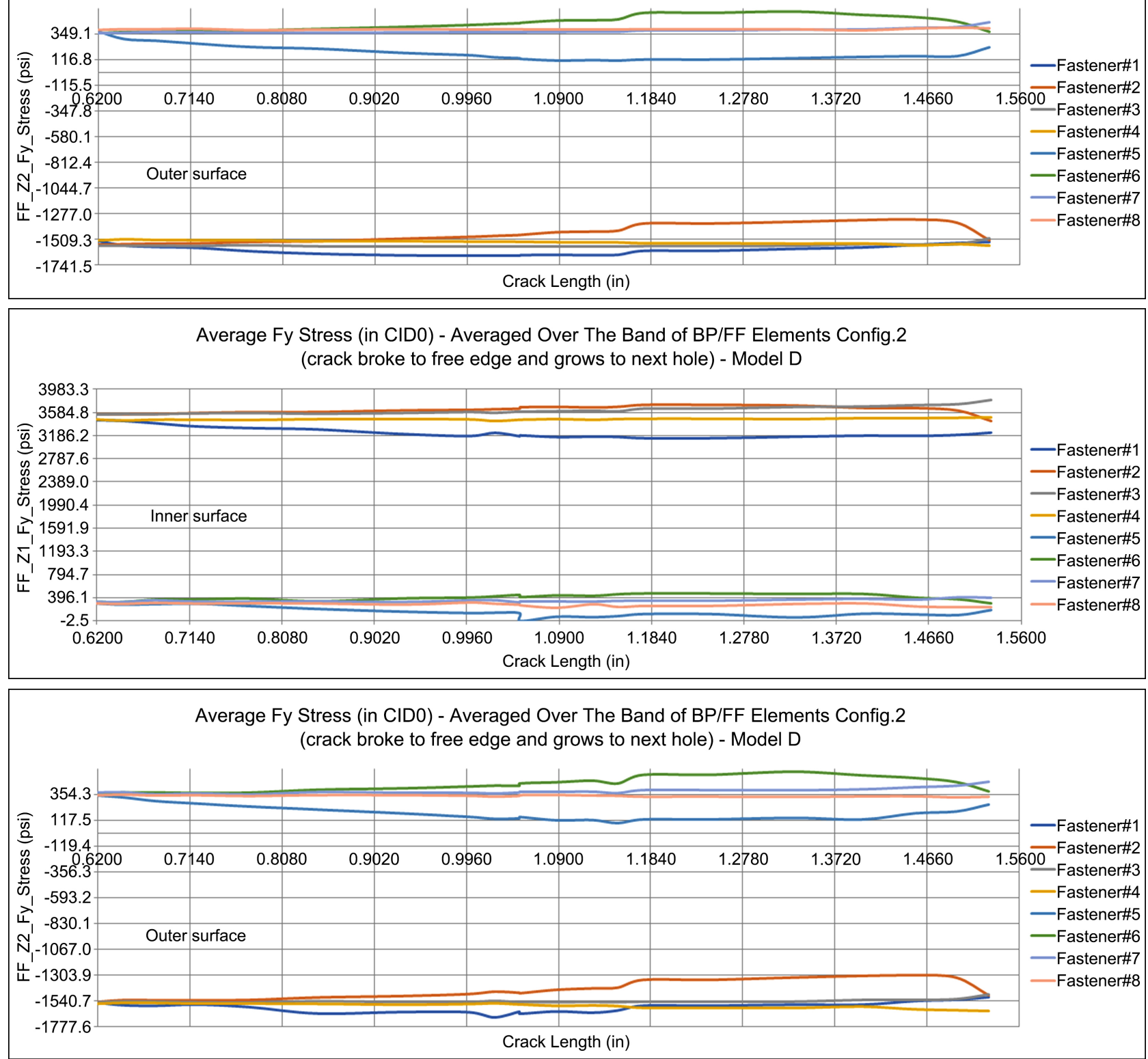

Figure 20. Distribution of strip wide averages of far-field stress on upper and lower surfaces as function of crack length in Config. 2. 
Average Fy Stress (in CID0) - Averaged Over The Band of BP/FF Elements Config.3

(crack grows left \& right from edge hole) - Model C

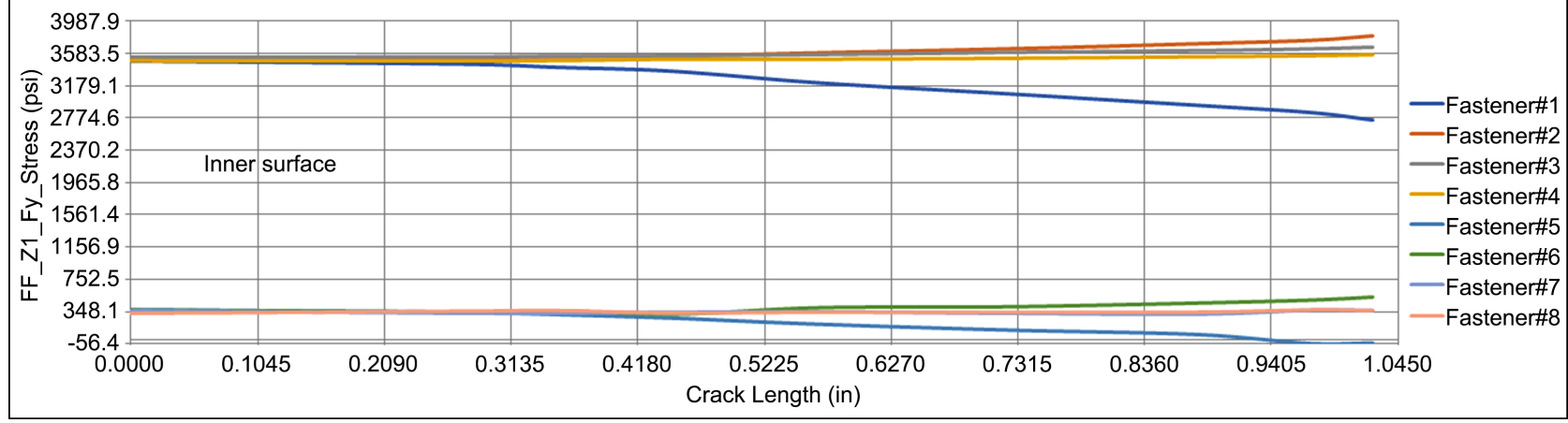

Average Fy Stress (in CID0) - Averaged Over The Band of BP/FF Elements Config.3

(crack grows left \& right from edge hole) - Model C
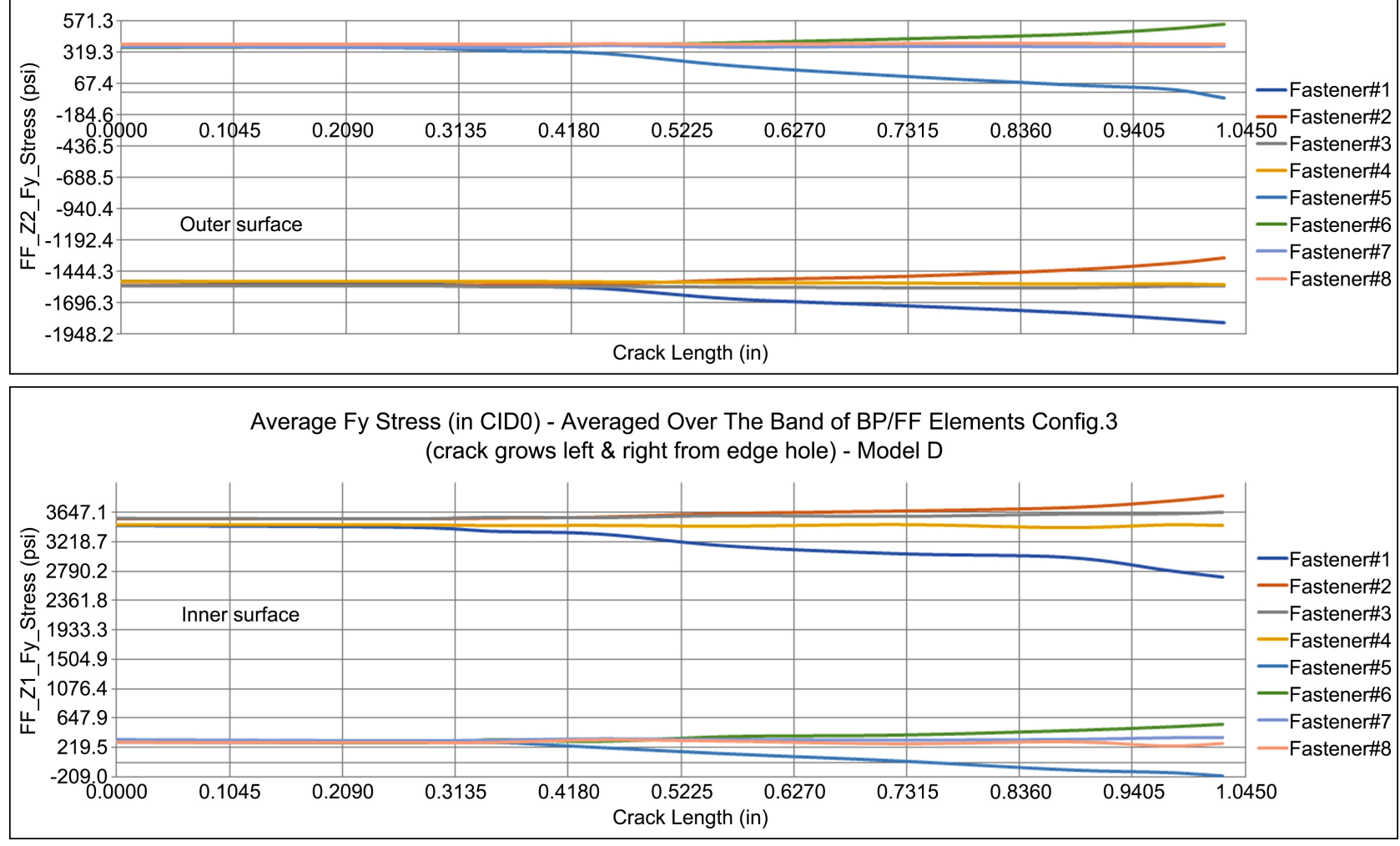

Average Fy Stress (in CID0) - Averaged Over The Band of BP/FF Elements Config.3

(crack grows left \& right from edge hole) - Model D

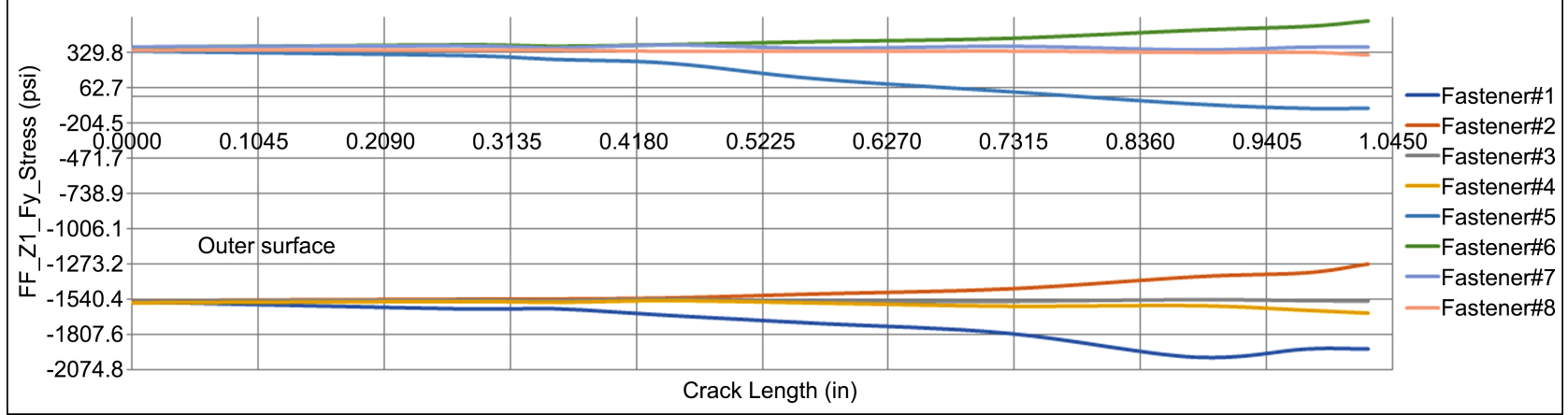

Figure 21. Distribution of strip wide averages of far-field stress on upper and lower surfaces as function of crack length in Config. 3. 

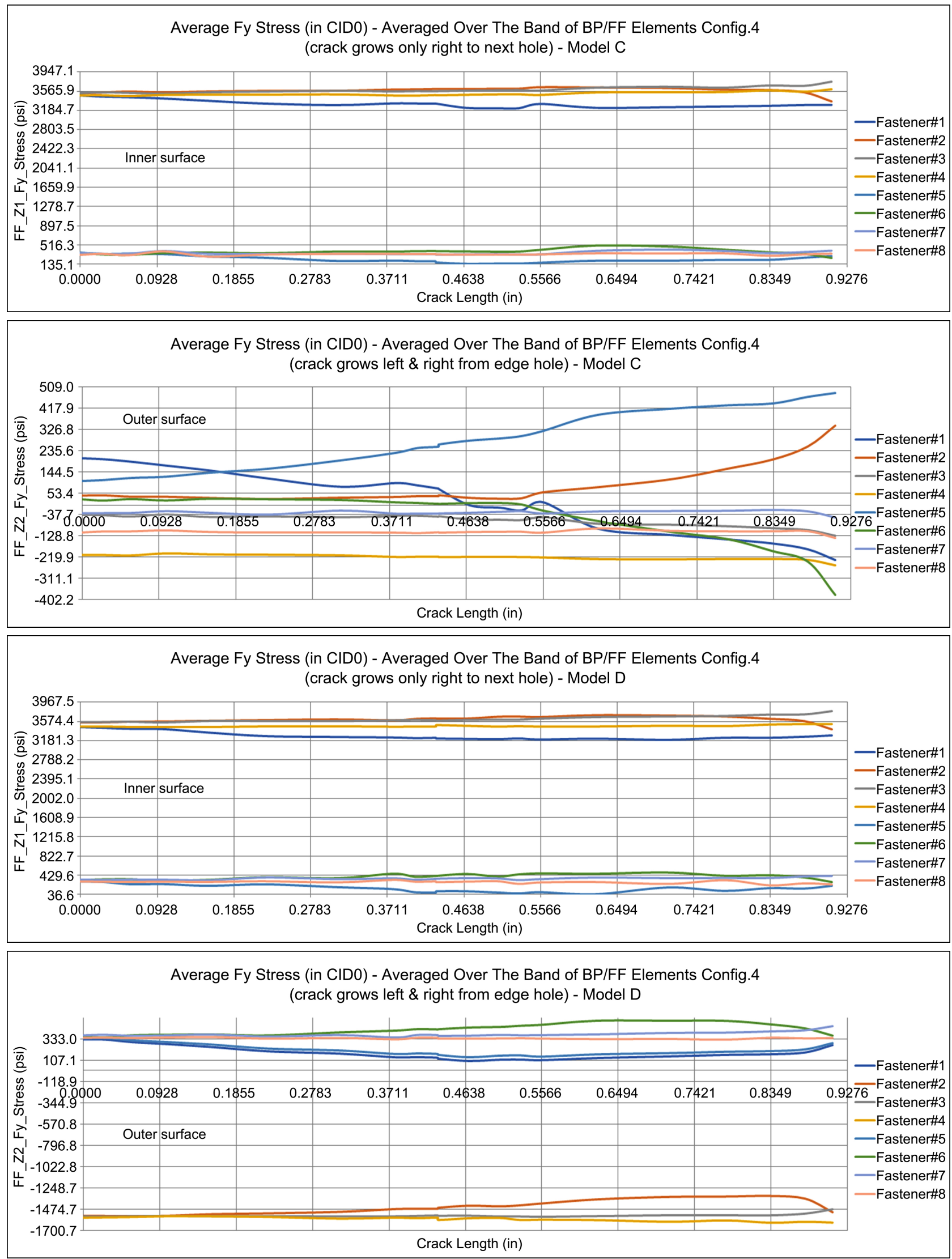

Figure 22. Distribution of strip wide averages of far-field stress on upper and lower surfaces as function of crack length in Config. 4 . 
Average Fy Stress (in CID0) - Averaged Over The Band of BP/FF Elements Config.5 (crack grows only left from scnd. hole to edge hole) - Model C

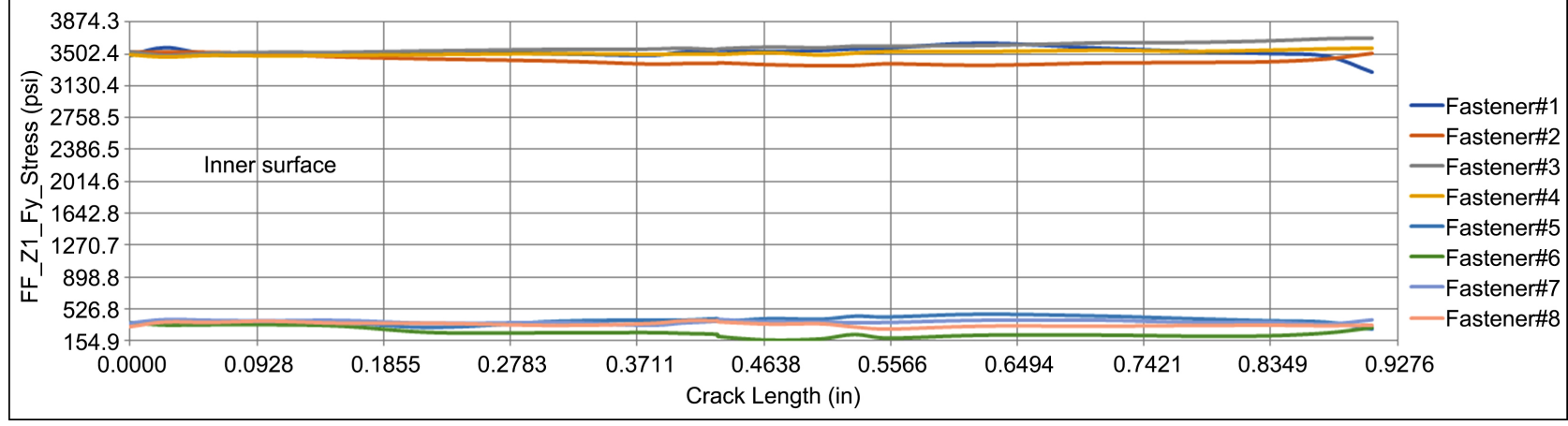

Average Fy Stress (in CID0) - Averaged Over The Band of BP/FF Elements Config.5 (crack grows left \& right from edge hole) - Model C
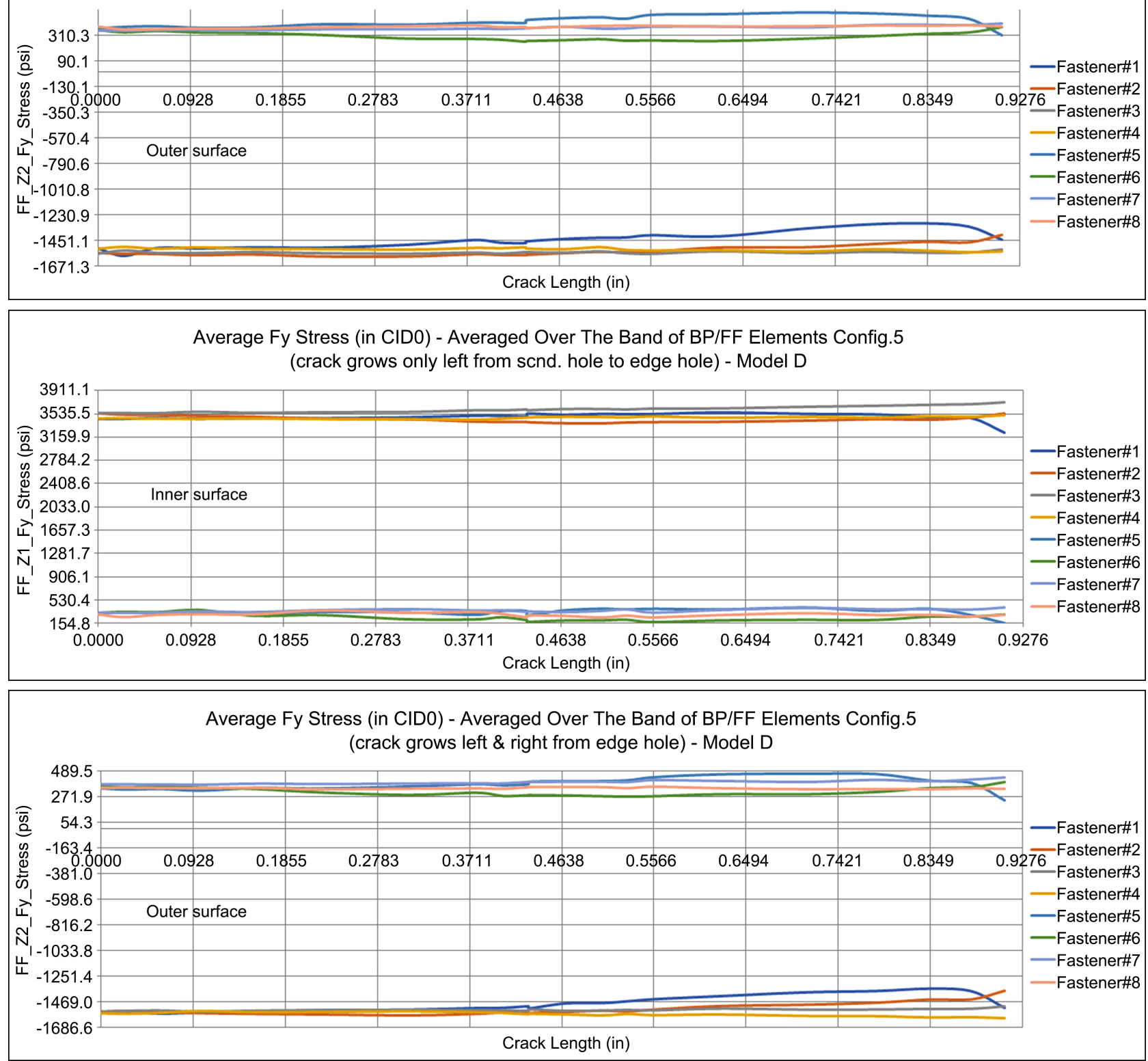

Figure 23. Distribution of strip wide averages of far-field stress on upper and lower surfaces as function of crack length in Config. 5. 
Table 7. Baseline "average" far-field stresses on a 0.455 " wide strip ahead of the fasteners for the inner surface of model C Below max-min differences between the elements in the strip.

\begin{tabular}{|c|c|c|c|c|c|c|c|c|c|}
\hline \multicolumn{10}{|c|}{$\begin{array}{l}\text { Baseline Surface Z1 FarField "Average" Stresses Ahead of Fasteners Holes } \\
\text { (evaluated over 2.3D, symmetrical about fast } \mathrm{C} / \mathrm{L} \text { ) And The Bands of Variations }\end{array}$} \\
\hline Model & & Pin/Hole & Pin/Hole & Pin/Hole & Pin/Hole & Pin/Hole & Pin/Hole & Pin/Hole & Pin/Hole \\
\hline Panel & P1 & 1 & 2 & 3 & 4 & 5 & 6 & 7 & 8 \\
\hline Hole Dia & (in) & 0.1895 & 0.1895 & 0.1895 & 0.1895 & 0.1895 & 0.1895 & 0.1895 & 0.1895 \\
\hline Crack Length@ Current Fastener & (in) & 0.000000 & 0.000000 & 0.000000 & 0.000000 & 0.000000 & 0.000000 & 0.000000 & 0.000000 \\
\hline Stress Fx ('load' type equivalent - \#1) & $(p s i)$ & 946.71 & 1197.16 & 1210.24 & 962.53 & 219.33 & 195.63 & 205.37 & 229.01 \\
\hline Stress Fy $(\ldots \# 1)$ & $(p s i)$ & 3483.94 & 3520.40 & 3530.87 & 3488.06 & 366.34 & 363.62 & 358.34 & 319.31 \\
\hline Stress Fxy (median value - \#2) & $(p s i)$ & -211.96 & -42.29 & 39.33 & 219.10 & -129.95 & -22.78 & 25.06 & 141.40 \\
\hline Stress MaxPrp (median value $-\# 3$ ) & $(p s i)$ & 3499.86 & 3525.03 & 3535.45 & 3505.11 & 442.81 & 369.78 & 365.99 & 423.90 \\
\hline Stress MinPrp $(\ldots \# 3)$ & $(p s i)$ & 934.98 & 1192.52 & 1205.66 & 951.01 & 143.39 & 189.46 & 196.76 & 121.89 \\
\hline Stress MaxShear(...\#1) & $(p s i)$ & 1749.93 & 1762.51 & 1767.72 & 1752.56 & 221.40 & 184.89 & 183.00 & 211.95 \\
\hline \multicolumn{10}{|c|}{ \#1 using SUMPROD(El_Strs*El_Side)/SUM(El_Side) } \\
\hline \multicolumn{10}{|l|}{ \#2 MEDIAN(all elems. considered) } \\
\hline \multicolumn{10}{|c|}{ \#3 prp strs for each element and then used MEDIAN of all elems. considered } \\
\hline \multicolumn{10}{|c|}{ Variation between min and max values } \\
\hline Hole No & & 1 & 2 & 3 & 4 & 5 & 6 & 7 & 8 \\
\hline delta Fx & $(p s i)$ & 236.68 & 159.80 & 155.66 & 241.75 & 81.42 & 86.39 & 93.74 & 80.68 \\
\hline delta Fy & $(p s i)$ & 61.79 & 55.45 & 53.79 & 63.33 & 128.47 & 135.00 & 137.46 & 132.92 \\
\hline delta Fxy & $(p s i)$ & 314.88 & 281.57 & 282.40 & 320.68 & 22.45 & 70.21 & 74.21 & 34.94 \\
\hline delta MaxPrp & $(p s i)$ & 97.10 & 57.59 & 56.30 & 100.23 & 90.77 & 141.83 & 145.44 & 84.13 \\
\hline delta MinPrp & $(p s i)$ & 276.30 & 173.30 & 169.00 & 283.05 & 47.68 & 93.23 & 101.71 & 42.40 \\
\hline delta MaxShear & $(p s i)$ & 48.55 & 28.80 & 28.15 & 50.12 & 45.39 & 70.92 & 72.72 & 42.06 \\
\hline
\end{tabular}


Table 8. Baseline "average" far-field stresses on a 0.455 " wide strip ahead of the fasteners for the inner surface of model C Below max-min differences between the elements in the strip.

\begin{tabular}{|c|c|c|c|c|c|c|c|c|c|}
\hline \multicolumn{10}{|c|}{$\begin{array}{l}\text { Baseline Surface Z2 FarField "Average" Stresses Ahead of Fasteners Holes } \\
\text { (evaluated over 2.3D, symmetrical about fast } \mathrm{C} / \mathrm{L} \text { ) And The Bands of Variations }\end{array}$} \\
\hline Model & & Pin/Hole & Pin/Hole & Pin/Hole & Pin/Hole & Pin/Hole & Pin/Hole & Pin/Hole & Pin/Hole \\
\hline Panel & $\mathrm{P} 1$ & 1 & 2 & 3 & 4 & 5 & 6 & 7 & 8 \\
\hline Hole Dia & (in) & 0.1895 & 0.1895 & 0.1895 & 0.1895 & 0.1895 & 0.1895 & 0.1895 & 0.1895 \\
\hline Crack Length@ Current Fastener & (in) & 0.000000 & 0.000000 & 0.000000 & 0.000000 & 0.000000 & 0.000000 & 0.000000 & 0.000000 \\
\hline Stress Fx ('load' type equivalent - \#1) & $(p s i)$ & -276.30 & -526.02 & -538.00 & -287.94 & 241.77 & 277.16 & 267.92 & 245.11 \\
\hline Stress Fy $(\ldots \# 1)$ & $(p s i)$ & -1524.90 & -1560.14 & -1566.67 & -1523.35 & 375.11 & 357.14 & 359.08 & 387.60 \\
\hline Stress Fxy (median value - \#2) & $(p s i)$ & 202.95 & 42.40 & -41.29 & -210.61 & 107.34 & 27.42 & -32.23 & -114.24 \\
\hline Stress MaxPrp (median value $-\# 3$ ) & $(p s i)$ & -255.81 & -519.67 & -531.61 & -264.74 & 467.11 & 462.96 & 464.86 & 477.22 \\
\hline Stress MinPrp $(\ldots \# 3)$ & $(p s i)$ & -1556.42 & -1567.72 & -1573.97 & -1556.94 & 162.06 & 176.24 & 167.45 & 167.64 \\
\hline Stress MaxShear(...\#1) & $(p s i)$ & 778.21 & 783.86 & 786.98 & 778.47 & 233.56 & 231.48 & 232.43 & 238.61 \\
\hline \multicolumn{10}{|c|}{ \#1 using SUMPROD(El_StrsEl_Side)/SUM(El_Side) } \\
\hline \multicolumn{10}{|l|}{ \#2 MEDIAN(all elems. considered) } \\
\hline \multicolumn{10}{|c|}{ \#3 prp strs for each element and then used MEDIAN ovel all elems. considered } \\
\hline \multicolumn{10}{|c|}{ Variation between min and max values } \\
\hline Hole No & & 1 & 2 & 3 & 4 & 5 & 6 & 7 & 8 \\
\hline delta Fx & $(p s i)$ & 212.93 & 137.91 & 135.85 & 218.70 & 82.28 & 62.00 & 68.88 & 75.25 \\
\hline delta Fy & $(p s i)$ & 9.13 & 15.53 & 10.67 & 10.77 & 136.51 & 133.35 & 135.64 & 141.15 \\
\hline delta Fxy & $(p s i)$ & 247.38 & 218.73 & 221.14 & 253.89 & 431.94 & 416.21 & 429.93 & 428.89 \\
\hline delta MaxPrp & $(p s i)$ & 264.69 & 157.59 & 155.70 & 274.25 & 333.42 & 284.88 & 293.16 & 339.41 \\
\hline delta MinPrp & $(p s i)$ & 73.23 & 13.68 & 16.41 & 76.78 & 287.17 & 216.41 & 226.40 & 280.35 \\
\hline delta MaxShear & $(p s i)$ & 36.61 & 6.84 & 8.21 & 38.39 & 182.23 & 142.44 & 146.58 & 181.45 \\
\hline
\end{tabular}


Very significant variations in the bypass stresses as cracks grow were observed after the cracked hole and the adjacent fastener. Exemplifications of these variations are shown for cracks at fastener 1 in Figure 24 for single tip crack and in Figure 25 for cracks with two tips. These large variations are due mainly to the variations in fastener loads with effects of secondary bending having a smaller contribution after the first row of fasteners.

The ranges of variation for max-min principal stress values are relatively similar to the stress components in Oy.

All the curves of variation can be converted to a non dimensional parametric format relatively easy once the secondary bending is incorporated distinctly and unambiguously.

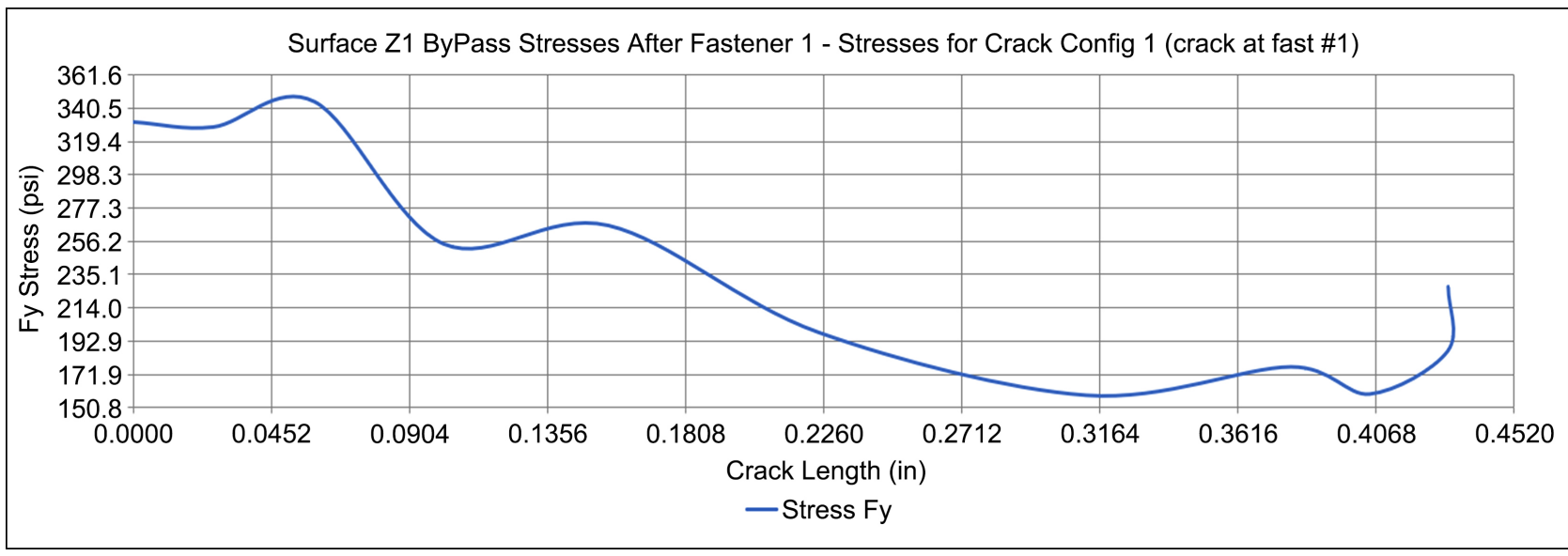

Surface Z1 ByPass Stresses After Fastener 1 - Stresses for Crack Config 1 (crack at fast \#1)

\begin{tabular}{|c|c|c|c|c|c|c|c|c|c|c|c|c|}
\hline & $\mathrm{C}$ & BaseLn & C1_L1 & C1_L2 & C1_L3 & C1_L4 & C1_L5 & C1_L6 & C1_L7 & C1_L8 & C1_L9 & C1_L10 \\
\hline & $\mathrm{P} 1$ & 0 & 1 & 2 & 3 & 4 & 5 & 6 & 7 & 8 & 9 & 10 \\
\hline Hole Dia & (in) & 0.1895 & 0.1895 & 0.1895 & 0.1895 & 0.1895 & 0.1895 & 0.1895 & 0.1895 & 0.1895 & 0.1895 & 0.1895 \\
\hline
\end{tabular}

Crack Length@ Current Fastener (in) 0.0000000 .0260400 .0592300 .1015500 .1555000 .2242700 .3119500 .3790000 .4047400 .4303000 .430480

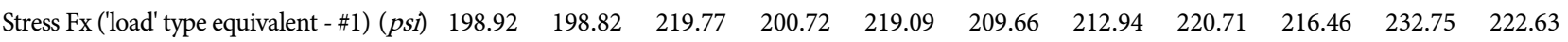

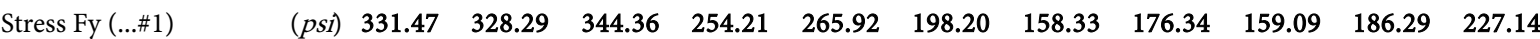

$\begin{array}{lllllllllllll}\text { Stress Fxy (median value - \#2) } & (p s i) & -130.48 & -123.46 & -147.88 & -145.87 & -166.71 & -204.64 & -250.32 & -308.40 & -339.71 & -386.02 & -228.56\end{array}$ Stress MaxPrp (median value - \#3) (psi) $\quad 414.98 \quad 404.95 \quad 438.50 \quad 370.87 \quad 395.03 \quad 420.31 \quad 443.69 \quad 507.96 \quad 539.03 \quad 622.92 \quad 445.47$

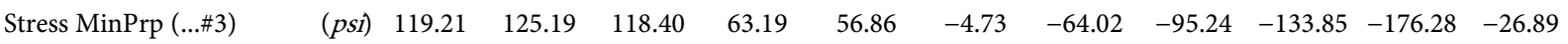

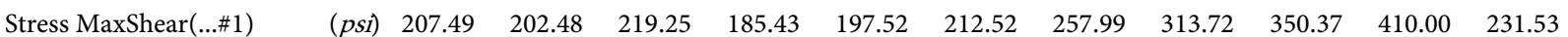
\#1 using SUMPROD(El_StrsEl_Side)/SUM(El_Side)

\#2 MEDIAN(all elems. considered)

\#3 prp strs for each element and then used MEDIAN of all elems. considered

Figure 24. Distribution of strip wide averages of bypass stress after fastener \#1 on the lower surfaces as function of crack length in Config. 1. 


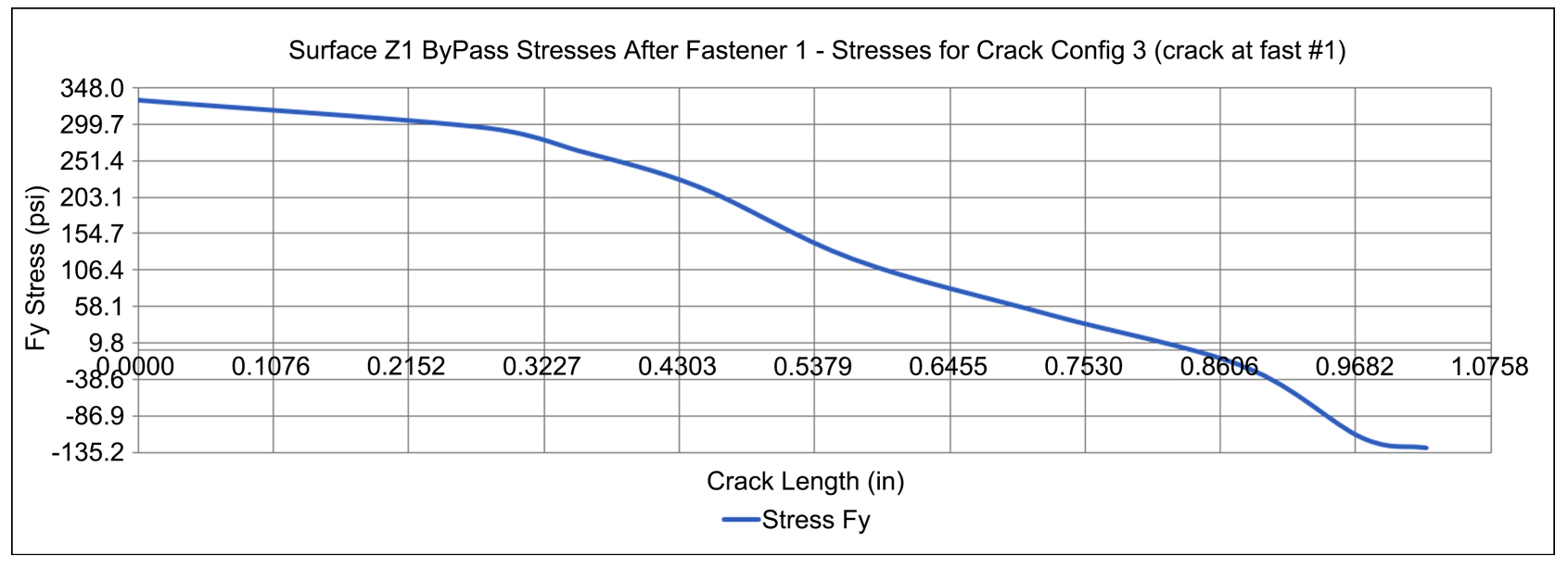

\begin{tabular}{|c|c|c|c|c|c|c|c|c|c|c|}
\hline \multicolumn{11}{|c|}{ Surface Z1 ByPass Stresses After Fastener 1 - Stresses for Crack Config 3 (crack at fast \#1) } \\
\hline & $\mathrm{C}$ & BaseLn & C3_R2L1 & C3_R3L2 & C3_R4L3 & C3_R5L4 & C3_R6L5 & C3_R7L6 & C3_R8L7 & C3_R9L8 \\
\hline & P1 & 0 & 1 & 2 & 3 & 4 & 5 & 6 & 7 & 8 \\
\hline Hole Dia & (in) & 0.1895 & 0.1895 & 0.1895 & 0.1895 & 0.1895 & 0.1895 & 0.1895 & 0.1895 & 0.1895 \\
\hline Crack Length@ Current Fastener & (in) & 0.000000 & 0.274770 & 0.350280 & 0.446550 & 0.569270 & 0.725720 & 0.880450 & 0.973240 & 1.024540 \\
\hline $\begin{array}{l}\text { Stress Fx ('load' type equivalent - } \\
\# 1)\end{array}$ & $(p s i)$ & 198.92 & 205.45 & 212.13 & 224.27 & 244.96 & 269.23 & 274.77 & 253.07 & 245.97 \\
\hline Stress Fy $(\ldots \# 1)$ & $(p s i)$ & 331.47 & 295.20 & 264.55 & 216.48 & 120.80 & 47.28 & -21.63 & -115.17 & -128.78 \\
\hline Stress Fxy (median value - \#2) & $(p s i)$ & -130.48 & -141.69 & -145.32 & -162.11 & -155.56 & -183.20 & -204.63 & -212.69 & -267.58 \\
\hline Stress MaxPrp (median value -\#3) & $(p s i)$ & 414.98 & 399.38 & 387.06 & 365.57 & 382.76 & 391.38 & 396.39 & 370.03 & 384.51 \\
\hline Stress MinPrp (...\#3) & $(p s i)$ & 119.21 & 94.08 & 75.40 & 26.29 & -24.98 & -120.74 & -205.94 & -283.34 & -344.71 \\
\hline Stress MaxShear $(\ldots \# 1)$ & $(p s i)$ & 207.49 & 199.69 & 193.53 & 182.79 & 203.87 & 256.06 & 301.16 & 326.68 & 366.12 \\
\hline \multicolumn{11}{|c|}{ \#1 using SUMPROD(El_Strs*El_Side)/SUM(El_Side) } \\
\hline \multicolumn{11}{|l|}{ \#2 MEDIAN(all elems. considered) } \\
\hline$\# 3$ prp strs for each element and th & ten us & MEDIA & of all eler & conside & & & & & & \\
\hline
\end{tabular}

Figure 25. Distribution of strip wide averages of bypass stress after fastener \#1 on the lower surfaces as function of crack length in Config. 3.

\section{Discussion}

Besides cold working, the parameter with the most influence on the fatigue strength is the fastener fit. A close tolerance fit that makes the fastener interfere with the deformations at the hole, reduces the local stress variations at the hole when subjected varying external loads. When the clearance increases, the interference of the fastener with the hole deformations is reduced and larger stress variations precipitate fatigue damage. Similarly, when cracks are developed at the hole, under load the interference with the fastener is reduced, there are moderate reductions in the load transfer at that fastener but, for single shear joints, the fastener tilt increases causing an increase in the hole stresses near the plate surfaces. At the same time there is an increase in the loads transferred at 
the adjacent fastener holes that affect their baseline fatigue strength.

The results for the fasteners load transfer function show that regardless of cracking configuration, at the cracked hole the load decreases by up to $20 \%$ of its value in non-cracked conditions and that, after the ligament fracture, the fastener continues to transfer that load until new cracks develop and grow. As the load transfer decreases at the cracked hole, the increases at the adjacent holes are up to $10 \%-12 \%$. It is especially noticeable that after breakage of the free edge ligament, the load at that fastener returns almost to its baseline value (the "hook effect").

These results also showed small but realistic differences in the fastener load transfer functions for the two different sets of plate lateral restraints, which make them very suitable for laboratory testing and FEA calibrations. This analysis accounted for the bolt flexibility and tilt, but the effects of friction have not been included in this stage, as friction is traditionally neglected from the structural calculations during design and sizing. Although very significant for the fatigue strength of the plate near the hole, apart for their influence on friction, the clamping forces are not expected to have any other significant effect on the fasteners load transfer in either baseline and cracked configurations. For laboratory testing of the load transfer functions with instrumented bolts, the effects of friction can be minimized by oil lubrication between the faying surfaces.

The through thickness distribution of the bearing loads/bearing stresses at the fastener holes was found to have maximum values at the faying surface, and this fact is of practical importance for inaccessible single shear joints and for multi layered joints with high eccentricities in the load path, where the crack can grow undetected for long durations. These results are only preliminary until the more in depth work on the effects of clamp-up forces and friction is completed. In [2] it is stated that testing has shown clamping forces to have a pronounced effect on the fatigue life of joints ${ }^{10}$. The clamping forces appear to be also a main factor in the eye-brow cracking around the periphery of fastener heads that was observed and reported for in-service aircraft.

The results for secondary bending have been found to be potentially very significant for the fatigue strength of the joint with secondary bending ratios $\sigma_{\text {bend }} / \sigma_{\text {tens }}=2.5$ at a distance of two hole diameters ahead of the holes ${ }^{11}$. As the main scope of these analytical investigations was to establish load transfer functions for the fasteners after cracking occurs, the externally applied stress was un-

\footnotetext{
${ }^{10}$ The use of ovalized "self locking" nuts with standard csk. bolts installed in clearance fit holes produced less than $20 \%$ of the normal life of a two row single shear joint, when torqued in a degreased condition compared with the specified lubrication. The cause of the short fatigue life was insufficient clamping.

In another test program (also spectrum testing) studying fatigue crack growth from $1 \mathrm{~mm}$ corner flaws made at the edge of fastener holes, the fatigue life increased by a factor of 4 when torqueing up the clearance fit bolts to the specifications.

${ }^{11}$ To correlate with the standardized location for strain gauge measurements for secondary bending and for optimum determination of the bypass forces by the integration method (AGARD working group - "Fatigue Rated Fastener Systems").
} 
itary and small $(1000 \mathrm{psi})$ to ensure that no plasticity occurs for the meshing scale used. It is possible that for more realistic tension stresses of $10-25 \mathrm{ksi}$, the secondary bending ratios might be smaller at that distance.

The far field and the bypass stresses were determined from the surface stresses (for use with the Tate \& Rosenfeld method) and show that the variations to crack length are strongly influenced by secondary bending effects An alternative approach to the integration method for laboratory determination of the far-field and bypass stresses, is proposed when using FEA simulations, by the average running bending moment of shell elements. The results for the bypass loads after the crack initiation show large differences from the $2 \mathrm{D}$ beam method for single shear joints.

Regardless of how the fastener loads in plate joints are established for baseline configurations (3D FEA modelling or by using fastener flexibilities), the laboratory test measurements show a pronounced 3-dimensional distribution Popular methods for determining the fastener loads and the far field bypass stresses are based on the beam analysis approach developed by Tate \& Rosenfeld ${ }^{12}$ which is 2-dimensional and therefore cannot capture the spatial distribution of the stresses in plates. The distribution of stresses in plates has high values close to the fasteners and sharp decays further away. The AGARD working group addressed this aspect by standardizing the number (to 8) and the locations of strain gauges for the laboratory determinations of the far field and by-pass stresses in fatigue rated fastener systems.

The curves shown are for 7075-T6 sheet metal (0.063" and 0.070" thick) joined with steel Hi-Lok fasteners installed in close tolerance fit holes. Most are shown in dimensional form, but they can be converted without any difficulty to any non-dimensional format of choice by division with constant values (eg. the crack length/hole diameter and load in cracked configuration/baseline load) and used as such or in the form of envelopes for at the cracked hole and at the adjacent hole with the highest fastener load transfers.

For other gauges, materials or fastener types they are expected to differ in values, but once verified and calibrated, the finite element approach may be used as a cost effective method of establishing parametric relationships between the crack length and the amounts of load transfer and bypass.

Popular fracture mechanics programs used are based on LFEM, because the structures are sized at design to operate within the elastic range of the materials. These programs account for the crack length through a geometry function largely known as "the beta function". Beta functions incorporate the stress variation in the net section at the crack, using as reference a clearly specified and constant far-field stress. In the case of growing cracks in a joint, the reference stress ahead of the hole (the far field stress) is changing as the fastener load is changing, and consequently the bypass load is changing. Some of the parameters that affect the fatigue strength have also a direct influence on the changes in the

\footnotetext{
${ }^{12}$ Tate \& Rosenfeld used this approach in 1946 to calculate the bypass force.
} 
reference stresses used.

With adequate knowledge of the baseline stress environment(s) and of the fasteners load transfer, the use of parametrized curves developed analytically (and calibrated by a small number of laboratory tests), may be used to adjust the spectrum stresses at every several blocks during the crack growth and residual strength analysis. The benefit will be in increased inspection intervals for the critical locations, without any reduction in the confidence levels for the results. In the design stage, they can be used for predictions on the appearance of multi-site fatigue damage at those details.

\section{Summary}

The aim of this work was to develop a method that enables a relatively quick and cost effective development of digitized curves for the variations in the fastener load transfer functions and to the far field and bypass running loads during crack growth and after the fracture of ligaments for single shear joints expected to see loading spectra with multiple amplitudes and mean stress values. For this purpose, the variation curves have been developed in terms of vectorial quantities (forces and moments) that are easy to visualize and interpret by most engineers. The conversions of the load curve to stresses in user preferred parametric formats are simple and can be done on most personal computers.

The use of these parametric curves to adjust the spectrum stress with multiple amplitude and mean values increases the accuracy of the predictions for crack growth and residual strength at the critical location, and translates into longer inspection intervals with no reduction in the confidence levels for the results.

The method proposed has the following characteristics:

- It is accounting for the pronounced 3-dimensional distribution of stresses in single shear joints with multiple rows of fasteners;

- It is applicable to cycles with bulk stresses in elastic range only;

- It accounts for installation clearance and for bolt tilting effects;

- It incorporates and quantifies the effects of secondary bending in a manner that is easy to interrogate and to verify by standard tests for fatigue rated joints;

- It provides quantified precision information on the averaged loads and stresses of the same nature;

- The computations and post processing are highly automated for analysis of different materials, plate thicknesses and fasteners configurations;

- All results are verifiable at all stages and the data reduction is shown in user friendly formats for interpretation and for engineering judgement;

- When used in conjunctions with calibration data from laboratory testing, it provides reliable data for design and sizing in new programs or for use in the specification of inspection intervals for structural integrity on existing platforms.

In addition to incorporating the effects of friction for easier calibration of the 
FEA to laboratory testing results, the effect of the crack length when the joint is loaded in shear needs to be also quantified in further studies.

\section{Acknowledgements}

Many thanks to my brother Gabriel and to Erica Bossell for their assistance and advice with the CAD and the graphical work, to Kevin Watters and Michael Houston for their reviews, consultations and inputs and to all my colleagues from QinetiQ who assisted in various ways during this project.

I am also thanking the WFT Project Authority at the Australian Defence Science and Technology Organization for the permission to use an extract of their work as experimental evidence for some of the analytical results.

\section{Conflicts of Interest}

The author declares no conflicts of interest regarding the publication of this paper.

\section{References}

[1] Peridas, G., Korsunsky, A.M. and Hills, D.A. (2003) The Relationship between the Dang Van Criterion and the Traditional Bulk Fatigue Criteria. The Journal of Strain Analysis for Engineering Design, 38, 201-206.

http://journals.sagepub.com/doi/abs/10.1243/030932403765310536?journalCode=sd 迆

https://doi.org/10.1243/030932403765310536

[2] Piotrowski, D. and Bode, M. (2006) NDT Comparison of In-Service Cracks, Manufactured Cracks and EDM Notches. Quantitative Nondestructive Evaluation Conference, Portland, 31 July-4 August 2006. https://www.osti.gov/servlets/purl/1264593 https://doi.org/10.1063/1.2718189

[3] Harris, C.E., Piascik, R.S. and Newman Jr., J.C. (2020) A Practical Engineering Approach to Predicting Fatigue Crack Growth in Riveted Joints. https://ntrs.nasa.gov/archive/nasa/casi.ntrs.nasa.gov/20040086665.pdf

[4] Yu, D.Z., Liu, W.L., Wang, L. and Xu, L. (2018) Experimental Study on Fatigue Life of Wide Bolted Lap Joint. 2018 International Conference on Power, Energy and Environmental Engineering, 184-188.

http://dpi-proceedings.com/index.php/dteees/article/viewFile/23369/23005

[5] Rans, C.D., Alderliesten, R.CV. and Straznicky, P.V. (2009) Assessing the Effects of Riveting Induced Residual Stresses on Fatigue Crack Behaviour in Lap Joints by Means of Fractography. International Journal of Fatigue, 31, 300-308.

https://doi.org/10.1016/j.ijfatigue.2008.08.005 http://calvinrans.com/wp-content/uploads/2013/10/Rans2009-Int-J-Fatigue.pdf

[6] Jarfall, L.E. (1983) Shear Loaded Fasteners Installations, SAAB-Scania, Report KH $\mathrm{R}-3360$.

[7] Jarfall, L.E. (1967) Optimum Design of Joints: The Stress Severity Factor Concept. The Aeronautical Research Institute of Sweden.

[8] ESDU 85021 (1985) Analysis of Pretensioned Bolted Joints Subject to Tensile (Separating) Forces.

[9] Niu, M.C. (1997) Airframe Structural Design. Conmilit Press Ltd.. 
[10] ESDU FAT E.05.01 (1977) Endurance of Structural Joints (Aluminium Alloy Material-Tensile Loading).

[11] ESDU 89046 (1989) Fatigue of Aluminium Alloy Joints with Various Fastener Systems. Low Load Transfer.

[12] ESDU 90009 (1990) Fatigue of Aluminium Alloy Joints with Various Fastener Systems. Medium Load Transfer.

[13] ESDU 90018 (1990) Fatigue of Aluminium Alloy Joints with Various Fastener Systems. High Load Transfer.

[14] MSC Nastran (2019) Nonlinear (SOL 400) User Guide. Feature Pack1, 610-623.

[15] Gudas, C. (2019) Nastran SOL400 Modelling of Pre-Tensioned Fasteners in Assemblies with Large Deformations, MSC Indo-Pacific User Conference, Singapore, Sept.

[16] Swift, T. (1974) The Effects of Fastener Flexibility and Stiffener Geometry on the Stress Intensity in Stiffened Cracked Sheet. In: Prospects of Fracture Mechanics, Noordhoff International Publishing, Leyden, 419-436.

https://doi.org/10.1007/978-94-010-2314-6_25 


\section{Terminology}

BCTABL1 Nastran entry used to define a contact table

CAD Computer aided design

Fastener flexibility Associates its displacement to the unit force of fastener load and does not imply that fastener flexibility would be a constant

MPC Multi Point Constraint in Nastran is the most general-purpose way to define motion based relationships between the grids in the model. The motion of any degree of freedom at the dependent grid is defined linearly by the motions at any $n>1$ independent grids.

PCL Patran Control Language

RBE3 A Nastran non rigid interpolation element which establishes the motion at a dependent grid as the weighted average of the motion(s) at a set of independent grids. If used correctly the RBE3 does not add stiffness to the structure

\section{Notations}

$f_{b y} \quad$ Oy component of the total bearing load at the pin-hole interface

$\mathrm{f}_{x} \quad$ Running load in Ox direction

$\mathrm{f}_{y} \quad$ Running load in Oy direction

ksi Kilopound per square inch

lbf Pound force

$\mathrm{q}_{x y} \quad$ Shear flow in direction Ox along an edge parallel with Oy

D Hole diameter

L Ligament length

$\mathbf{F}_{\text {Ox }} \quad$ Strip-wide average surface stress component in Ox direction

$\mathbf{F}_{\mathrm{Oy}} \quad$ Strip-wide average surface stress component in Oy direction

$\mathrm{MPa} \quad$ Megapascal

N Newton

\section{Abbreviations}

$\begin{array}{ll}\text { BP } & \text { Bypass } \\ \text { FEA } & \text { Finite Element Analysis } \\ \text { FF } & \text { Far field } \\ \text { LEFM } & \text { Linear Elastic Fracture Mechanicsksi } \\ \text { LHS } & \text { Left hand side } \\ \text { RHS } & \text { Right hand side } \\ \text { WFT } & \text { Wing Fatigue Testing }\end{array}$

